\title{
PET Reconstruction with non-Negativity Constraint in Projection Space: Optimization Through Hypo-Convergence
}

\author{
Alexandre Bousse*, Matias Courdurier*, Élise Émond, Kris Thielemans, Senior Member, IEEE, \\ Brian F. Hutton, Senior Member, IEEE, Pablo Irarrazaval, Senior Member, IEEE, Dimitris Visvikis, Senior \\ Member, IEEE
}

\begin{abstract}
Standard positron emission tomography (PET) reconstruction techniques are based on maximum-likelihood (ML) optimization methods, such as the maximum-likelihood expectation-maximization (MLEM) algorithm and its variations. Most of these methodologies rely on a positivity constraint on the activity distribution image. Although this constraint is meaningful from a physical point of view, it can be a source of bias for low-count/high-background PET, which can compromise accurate quantification. Existing methods that allow for negative values in the estimated image usually utilize a modified loglikelihood, and therefore break the data statistics. In this work we propose to incorporate the positivity constraint on the projections only, by approximating the (penalized) log-likelihood function by an adequate sequence of objective functions that are easily maximized without constraint. This sequence is constructed such that there is hypo-convergence (a type of convergence that allows the convergence of the maximizers under some conditions) to the original log-likelihood, hence allowing us to achieve maximization with positivity constraint on the projections using simple settings. A complete proof of convergence under weak assumptions is given. We provide results of experiments on simulated data where we compare our methodology with the alternative direction method of multipliers (ADMM) method, showing that our algorithm converges to a maximizer which stays in the desired feasibility set, with faster convergence than ADMM. We also show that this approach reduces the bias, as compared with MLEM images, in necrotic tumors-which are characterized by cold regions surrounded by hot structures-while reconstructing similar activity values in hot regions.
\end{abstract}

Index Terms-PET Imaging, Penalized Maximum-Likelihood Image Reconstruction, Constrained Optimization, HypoConvergence.

This work was partially funded by Fondecyt, Grant Number 1141189 and Conicyt-PIA-Anillo ACT1416. Asterisk indicates corresponding authors.

*A. Bousse was with the Institute of Nuclear Medicine, University College London, London NW1 2BU, UK. He is now with LaTIM, INSERM, UMR 1101, Université de Bretagne Occidentale, Brest, France (email: alexandre.bousse@univ-brest.fr).

* M. Courdurier is with Departamento de Matemática, Pontificia Universidad Católica de Chile, Santiago, Chile (email: mcourdurier@mat.uc.cl).

É. Émond, K. Thielemans and B. F. Hutton are with the Institute of Nuclear Medicine, University College London, London NW1 2BU, UK.

B. F. Hutton is also with the Centre for Medical Radiation Physics, University of Wollongong, Wollongong NSW 2522, Australia.

P. Irarrazaval is with Departamento de Ingeniería Eléctrica and Instituto de Ingeniería Biológica y Médica, Pontificia Universidad Católica de Chile, Santiago, Chile.

D. Visvikis is with LaTIM, INSERM, UMR 1101, Université de Bretagne Occidentale, Brest, France.

\section{INTRODUCTION}

$\mathbf{U}$ NTIL the 1980's, tomographic image reconstruction, which includes positron emission tomography (PET), relied on filtered-backprojections (FBP), consisting in computing the generalized inverse of the Radon transform [1]. As an inverse of an operator between two functional spaces, FBP does not include a non-negativity assumption on the image, but only computes an image that matches the observed projection data. As a result of the direct inversion, high frequencies in the data are amplified by the ramp filter present in the FBP operator.

With the publication of the iterative maximum-likelihood expectation-maximization (MLEM) algorithm for emission tomography [2], [3] and its accelerated version [4], PET image reconstruction by maximum-likelihood (ML) techniques grew in popularity and replaced FBP in most clinical settings. By accounting for the statistical model of the emission data, MLEM delivers images with better noise and resolution properties, which can also be further controlled with the addition of a penalty term on the log-likelihood [5].

The MLEM algorithm consists in maximizing the expected log-likelihood, where the "expectation" is obtained from the activity estimate at the previous iteration. The underlying model assumes that the emissions at each voxel follow a Poisson distribution centered on the activity image. Hence, each MLEM iteration produces positive images. More generally, most iterative reconstruction algorithms impose positivity on the reconstructed image [5]-[7].

The drawback of the positivity constraint is the induced bias in low-activity (cold) regions. This bias is further amplified when the regions are surrounded by high-activity (hot) regions, which artificially increase the activity in cold regions due to spillover (partial volume effects). This compromises quantitative studies such as kinetic modeling or tumor texture analysis in the presence of hypoxia or necrosis [8], [9]. Moreover, iterative reconstruction algorithms suffer from noise-induced bias, which affects quantification in low-statistic scans with high-background (random and scatter) fraction [10].

Several approaches allowing for negative activity values have been proposed in order to reduce bias, including modifications of the original MLEM algorithm to impose an upper-bound and a (negative) lower-bound [11], [12], as well as Poisson/Gaussian 
mixture models [13], [14]. However, these methods depend on some parameters (lower-bound for [11], [12], likelihood mixture parameter for [13], [14]) which affect the reconstructed image characteristics. Therefore, these parameters need to be optimally chosen. In addition, these methods break the statistical model and therefore are vulnerable to noise amplification. More recently, Lim et al. [15] have developed a penalized maximum-likelihood (PML) algorithm for PET that performs maximization of the penalized log-likelihood (PLL) with positivity constraint on the projections, by maximizing the PLL over its entire domain of definition. The optimization problem was reformulated as an augmented Lagrangian saddle point problem, which was solved with the help of an alternative direction method of multipliers (ADMM) [16]. Consequently, its implementation depends on the choice of the augmented Lagrangian weight.

In this paper we propose an alternative to [15] to solve the PML problem with positivity constraint on the projections. We approximate the PLL function by an adequate sequence of objective functions without constraint on feasibility. This sequence is designed such that it hypo-converges to the original PLL (see Definition 1). The overall algorithm consists of approximately maximizing each objective function of the sequence successively and without constraint; the hypo-convergence of the sequence of objective functions allows the sequence of maximizers to converge to the PML with positivity constraint on the projections.

A preliminary version of this work was presented at the 2018 Nuclear Science Symposium and Medical Imaging Conference [17]. We substantially extended this work by incorporating a mathematical analysis of our algorithm and a complete proof of convergence (Section II). We also added a comparison with the ADMM method proposed by Lim et al. [15] on simulated data (Section III-C), as well as a quantitative analysis on simulated oncology images with a necrotic tumor (Section III-D).

\section{THEORY}

\section{A. PET Measurement}

The radiotracer activity distribution to be reconstructed takes the form of a digital image vector $\boldsymbol{f}=\left[f_{1}, \ldots, f_{n_{\mathrm{v}}}\right]^{\top} \in$ $\mathbb{R}^{n_{\mathrm{v}}}$, where $n_{\mathrm{v}}$ is the number of voxels in the volume and ' $T$, is the matrix transpose symbol. The activity at voxel $j$ (in $\mathrm{kBq}$ per unit of volume) is $[\boldsymbol{f}]_{j}=f_{j}$, the symbol $[\cdot]_{j}$ representing the $j$-th element of a vector (or a matrix if double indexation). The activity image is indirectly observed through the PET imaging system comprising $n_{\mathrm{b}}$ detector bins modeled by a system matrix $\boldsymbol{H} \in \mathbb{R}_{+}^{n_{\mathrm{b}} \times n_{\mathrm{v}}}$, where each entry $[\boldsymbol{H}]_{i, j}$ represents the probability (up to a scale) that a photon pair resulting from an annihilation in the $j$-th voxel is detected by the $i$-th detector bin. The system matrix accounts for the scanner geometry, sensitivity, acquisition time and resolution as well as the attenuation factors. The number of detected photon pairs at each bin is a random vector $\boldsymbol{g}=\left[g_{1}, \ldots, g_{n_{\mathrm{b}}}\right]^{\top} \in \mathbb{N}^{n_{\mathrm{b}}}$ that follows a Poisson distribution with independent entries,

$$
\forall i=1, \ldots, n_{\mathrm{b}}, \quad g_{i} \sim \operatorname{Poisson}\left(\bar{g}_{i}(\boldsymbol{f})\right) .
$$

The parameter $\bar{g}_{i}(\boldsymbol{f})$ represents the expected counts at bin $i$ and is defined as

$$
\forall i=1, \ldots, n_{\mathrm{b}}, \quad \bar{g}_{i}(\boldsymbol{f}) \triangleq[\boldsymbol{H} \boldsymbol{f}]_{i}+r_{i},
$$

where the $r_{i}$ 's represent the expected background events (randoms and scatter). The expected values $\bar{g}_{i}(\boldsymbol{f})$ and $r_{i} \geq 0$, $i=1, \ldots, n_{\mathrm{b}}$, are stored in vectors respectively denoted $\overline{\boldsymbol{g}}(\boldsymbol{f})$ and $\boldsymbol{r}$, so that (2) can be rewritten with the matrix formulation $\overline{\boldsymbol{g}}(\boldsymbol{f})=\boldsymbol{H} \boldsymbol{f}+\boldsymbol{r}$.

Remark 1. For the rest of the article we consider the following assumptions (see [7]). For $\boldsymbol{H} \in \mathbb{R}_{+}^{n_{\mathrm{b}} \times n_{\mathrm{v}}}$ we assume that $\min _{i}\left(\max _{j}\left([\boldsymbol{H}]_{i, j}\right)\right)>0$, i.e., no row of $\boldsymbol{H}$ is identically zero (there is no inane bin). For $\boldsymbol{g} \in \mathbb{N}^{n_{\mathrm{b}}}$ we assume that $\boldsymbol{g}^{\top} \boldsymbol{H} \mathbf{1}>0$, where $\mathbf{1}$ denotes a vector of ones, which is a sufficient condition for the strict concavity of the objective function in most settings, as demonstrated in [7]. Additionally, we will consider functions with values in $\overline{\mathbb{R}}=\mathbb{R} \cup\{-\infty\}$, in particular we extend the definition of $\log (x)$ to all $x \in \mathbb{R}$ considering $\log (x)=-\infty$ for $x \leq 0$. Finally, $\|\cdot\|$ denotes the $\ell^{2}$-norm.

\section{B. Penalized Maximum-Likelihood Reconstruction}

1) Poisson Distribution: Given a measurement $\boldsymbol{g}$, the likelihood of an image $\boldsymbol{f}$ is $\mathcal{L}(\boldsymbol{g} \mid \boldsymbol{f})=\prod_{i} p\left(g_{i} \mid \bar{g}_{i}(\boldsymbol{f})\right)$, with $p(n \mid \lambda)=\mathrm{e}^{-\lambda} \lambda^{n} / n$ !. Omitting the terms independent of $\boldsymbol{f}$, the $\log$-likelihood $L$ is

$$
\begin{aligned}
L(\boldsymbol{f}) & \triangleq \log \mathcal{L}(\boldsymbol{g} \mid \boldsymbol{f}) \\
& =\sum_{i=1}^{n_{\mathrm{b}}} h_{i}\left(\bar{g}_{i}(\boldsymbol{f})\right)
\end{aligned}
$$

where for all $i=1, \ldots, n_{\mathrm{b}}, h_{i}: \mathbb{R} \rightarrow \overline{\mathbb{R}}$ is defined as

$$
h_{i}(x) \triangleq \begin{cases}g_{i} \log (x)-x & \text { if } g_{i}>0 \text { and } x>0, \\ -x & \text { if } g_{i}=0 \text { and } x \geq 0, \\ -\infty & \text { otherwise. }\end{cases}
$$

The likelihood function $L$ is thereby finite on the subset $\mathcal{D} \subset$ $\mathbb{R}^{n_{\mathrm{v}}}$ defined as

$$
\begin{array}{r}
\mathcal{D} \triangleq\left\{\boldsymbol{f} \in \mathbb{R}^{n_{\mathrm{v}}}: \forall i \in\left\{1, \ldots, n_{\mathrm{b}}\right\}, \bar{g}_{i}(\boldsymbol{f}) \geq 0\right. \\
\text { and } \left.g_{i}>0 \Rightarrow \bar{g}_{i}(\boldsymbol{f})>0\right\} .
\end{array}
$$

which explicitly means each image $f$ (even with negative entries) is in $\mathcal{D}$ provided that $\bar{g}_{i}(\boldsymbol{f}) \geq 0$ and $\bar{g}_{i}(\boldsymbol{f})=0$ implies $g_{i}=0$, i.e., no annihilation is detected at bin $i$.

2) Standard Penalized Maximum-Likelihood with Positivity Constraint on the Image: Standard statistical image reconstruction in PET is formulated as the following constrained PML problem:

maximize $L(\boldsymbol{f})+U(\boldsymbol{f})$ subject to $\boldsymbol{f} \in \mathcal{D}$ and $\boldsymbol{f} \geq \mathbf{0}$.

where $U: \mathbb{R}^{n_{\mathrm{v}}} \rightarrow \mathbb{R}$ is a penalty term often defined as

$$
U(\boldsymbol{f})=-\gamma \sum_{j=1}^{n_{\mathrm{v}}} \sum_{m \in \mathcal{N}_{j}} \omega_{j, m} \psi\left(f_{j}-f_{m}\right),
$$


$\psi: \mathbb{R} \rightarrow \mathbb{R}^{+}$is assumed strictly convex, even, continuously differentiable (hence $\left.\lim _{|x| \rightarrow+\infty} \psi(x)=+\infty\right)^{1}, j \mapsto \mathcal{N}_{j} \subset$ $\left\{1, \ldots, n_{\mathrm{v}}\right\}$ is a connected neighboring system (i.e., for all pair $\left(j, j^{\prime}\right)$ there is a path $j=j_{0}, j_{1}, \ldots, j_{K}=j^{\prime}$ such that $j_{k} \sim j_{k+1}, \forall k=0, \ldots, K-1$, where here the ' $\sim$ ' symbol denotes the neighboring binary relation), $\omega_{j, m}=1 / \operatorname{dist}(j, m)$ and $\gamma$ is a penalty strength parameter.

The constraint in (5) is suitable to the definition of $f$, which represents a non-negative value that corresponds to the radioactivity at each voxel. It is also a convenient "box constraint" that can be enforced on each voxel independently. As a result, any optimization algorithm that ensures the $k$-th estimate $\boldsymbol{f}^{k}$ at iteration $k$ satisfies $\boldsymbol{f}^{k} \geq \mathbf{0}$ will provide, if it converges, a solution $\boldsymbol{f} \geq \mathbf{0}$ which satisfies the constraint $\mathcal{D}$.

A typical approach for ML and PML reconstruction in emission tomography is to utilize a sequence of suitable separable surrogate functions $Q\left(\cdot \mid \boldsymbol{f}^{k}\right)$ such that the new estimate $\boldsymbol{f}^{k+1}$ is obtained by maximization of $\boldsymbol{f} \mapsto Q\left(\boldsymbol{f} \mid \boldsymbol{f}^{k}\right)$. When $Q$ is a sum over the voxels $j$ of sub-functions depending on a single $[\boldsymbol{f}]_{j}$, the new estimate $\left[\boldsymbol{f}^{k+1}\right]_{j}$ is computed for each voxel $j$ independently. This class of method is particularly suitable for box constraints such as in (5). The surrogate functions $Q\left(\cdot \mid \boldsymbol{f}^{k}\right)$ can be for example the expected loglikelihood, as used for MLEM [2], [3], and the modified maximum-likelihood expectation-maximization (M-MLEM) [5] that includes a penalty term. It can also be a sum of paraboloid functions for each voxel [7], [18], [19]. Other methods, for instance using ordered subsets [4], one-step-late [6] and quasiNewton line-search [20], also rely on the non-negativity of $f$.

3) Penalized Maximum-Likelihood with Positivity Constraint on Projections: In a Poisson parameter estimation task, the positivity constraint is a source of bias which worsens with the presence of an additive constant term (background). Consider the following one-dimensional example: let $Y$ be a Poisson random variable of parameter $a x+b$, with $x, a, b>0$, and the task is to estimate $x$ from $Y$. The unconstrained maximumlikelihood estimator $\tilde{x}=(Y-b) / a$ is unbiased. On the other hand, the estimator with positivity constraint, $\hat{x}=\max (0,(Y-$ $b) / a$ ), has a strictly positive bias

$$
\begin{aligned}
\mathbb{E}[\hat{x}]-x & =\mathbb{E}\left[\frac{1}{a}(b-Y) \mathbf{1}_{[0, b[}(Y)\right] \\
& \geq \frac{b}{a} \mathbb{P}(\{Y=0\})=\frac{b}{a} \mathrm{e}^{-(a x+b)}>0
\end{aligned}
$$

where $\mathbf{1}_{A}$ denotes the indicator function of a set $A$. In addition, by noticing that $b-Y \leq b$ in (7), we observe that the bias is upper-bounded:

$$
\mathbb{E}[\hat{x}]-x \leq \frac{b}{a} .
$$

Therefore the bias is small whenever $b \ll a$. Otherwise, the estimator is more likely to suffer from a relatively larger bias as $x$ approaches 0 .

The above rationale translates to PET reconstruction, with $x$ corresponding to the activity image $f, a$ representing the true coincidence detection rate (multiplied by the acquisition time),

\footnotetext{
${ }^{1}$ For our experiments we used $\psi(x)=\frac{1}{2} x^{2}$
}

and $b$ corresponding to the expected background-event vector $\boldsymbol{r}$ (random and scatter). When the true coincidence fraction in the data vector $\boldsymbol{g}$ is high (relatively to the background), the constraint $\{\boldsymbol{f} \geq \mathbf{0}\}$ in (5) is not strongly enforced, since this condition fits reasonably well with the data. On the contrary, in a low-count/high-background situation (therefore low true coincidence fraction), a solution of (5) actively tries to cross the boundaries of $\{\boldsymbol{f} \geq \mathbf{0}\}$ in order to fit with the data, and the problem of noise-induced bias from such positivity constraint becomes more apparent in low-activity regions.

A solution is to replace the optimization problem (5) with

$$
\text { maximize } L(\boldsymbol{f})+U(\boldsymbol{f}) \text { subject to } \boldsymbol{f} \in \mathcal{D} \text {. }
$$

Solving (8) is less constraining than solving (5) as it allows for solutions with negative values, provided that they belong to $\mathcal{D}$. On the other hand, it is a challenging problem since the constraints are expressed through the system matrix $\boldsymbol{H}$, as opposed to coordinate-wise constraints.

Several methods [11]-[14] were proposed to allow for negative values in $\boldsymbol{f}$, but they do not solve (8). In addition, they depend on manually set parameters which impact on the reconstructed image properties (bias and noise). Recently, Lim et al. [15] developed a methodology to solve (8). They demonstrated that in a low-count/high-background situation their methodology provides better quantification than using the modified likelihood approach [13], [14] with a sub-optimal parameter. Their methodology was derived from an augmented Lagrangian formulation to separate the image $f$ from the projection $\boldsymbol{g}$ and to transfer the constraint to an auxiliary variable which does not involve the system matrix $\boldsymbol{H}$. The augmented Lagrangian obtained was then optimized with the help of an ADMM framework [16]. However, the speed of convergence of ADMM depends on the augmented Lagrangian parameter and therefore should be chosen appropriately (see [21], [22]).

\section{Proposed Approach}

1) Strategy: We propose an alternative to [15] to solve (8). Instead of directly maximizing $L$, we maximize a sequence of approximate $\log$-likelihood $L^{k}: \mathbb{R}^{n_{\mathrm{v}}} \rightarrow \mathbb{R}$ such that it converges to $L$ in an appropriate sense. We define $L^{k}$ as

$$
L^{k}(\boldsymbol{f})=\sum_{i=1}^{n_{\mathrm{b}}} h_{i}^{k}\left(\bar{g}_{i}(\boldsymbol{f})\right), \quad \boldsymbol{f} \in \mathbb{R}^{n_{\mathrm{v}}},
$$

with $h_{i}^{k}: \mathbb{R} \rightarrow \mathbb{R}$ defined as

$h_{i}^{k}(x)= \begin{cases}h_{i}\left(\varphi_{k}(x)\right)=g_{i} \log \varphi_{k}(x)-\varphi_{k}(x) & \text { if } \quad g_{i}>0 \\ \beta_{k} \log \varphi_{k}(x)-\varphi_{k}(x) & \text { if } \quad g_{i}=0\end{cases}$

and $\left.\varphi_{k}: \mathbb{R} \rightarrow\right] 0,+\infty[$ is an approximation of $x \mapsto \max (0, x)$ defined as

$$
\varphi_{k}(x) \triangleq \log \left(1+\exp \left(\alpha_{k} x\right)\right) / \alpha_{k},
$$

with $\alpha_{k} \rightarrow+\infty$ and $\beta_{k} \searrow 0^{+}$.

The concept of hypo-convergence is a key element in this article, and is defined in the following way. 
Definition 1. Consider the functions $F, F_{k}: \mathbb{R}^{n} \rightarrow \overline{\mathbb{R}}, k \in \mathbb{N}$. We say that $\left(F_{k}\right)_{k}$ hypo-converges to $F$, and we denote it as $F_{k} \stackrel{h}{\rightarrow} F$, if for all $\boldsymbol{x} \in \mathbb{R}^{n}$

(i) $\limsup F_{k}\left(\boldsymbol{x}_{k}\right) \leq F(\boldsymbol{x})$ for all $\quad \boldsymbol{x}_{k} \rightarrow \boldsymbol{x}$, and

(ii) $\underset{k}{\liminf } F_{k}\left(\boldsymbol{x}_{k}\right) \geq F(\boldsymbol{x})$ for some $\boldsymbol{x}_{k} \rightarrow \boldsymbol{x}$.

Remark 2. $F_{k} \stackrel{h}{\longrightarrow} F$ and $G_{k} \stackrel{h}{\rightarrow} G$ does not necessarily imply $F_{k}+G_{k} \stackrel{h}{\longrightarrow} F+G$. Moreover, $F_{k}=F$ for all $k$ does not imply $F_{k} \stackrel{h}{\longrightarrow} F$. It is however true if $F$ is upper semi-continuous (see Definition A.1). If $F_{k} \stackrel{h}{\rightarrow} F$ and $U$ is continuous then $F_{k}+U \stackrel{h}{\longrightarrow} F+U$.

When $\alpha_{k} \beta_{k} \rightarrow+\infty$, the sequence $\left(L^{k}\right)_{k}$ hypo-converges to $L$. This property is one of the main ingredients to demonstrate that maximizing $L+U$ can be achieved by maximizing $L^{k}+U$ sequentially (the equivalent notion for minimization problems is epi-convergence), as stated in the following theorem.

Theorem 1. Let $\boldsymbol{f}^{k}=\arg \max _{\boldsymbol{f} \in \mathbb{R}^{n_{\mathrm{v}}}} L^{k}(\boldsymbol{f})+U(\boldsymbol{f})$. Assume that $\alpha_{k} \beta_{k} \rightarrow+\infty$. Then

$$
\boldsymbol{f}^{k} \rightarrow \boldsymbol{f}^{\star}=\underset{\boldsymbol{f} \in \mathcal{D}}{\arg \max } L(\boldsymbol{f})+U(\boldsymbol{f}) .
$$

A complete proof is provided in Section II-D, with additional elements in Appendix A and B. In particular, we observe that under the mild conditions in Remark $1, L^{k}+U$ and $L+U$ have unique maximizers on $\mathbb{R}^{n_{\mathrm{v}}}$ and $\mathcal{D}$ respectively.

Remark 3. The choice (10) to approximate $h_{i}$ is non-unique and other approximations are possible. Another solution is the Poisson/Gaussian approximation introduced by Nuyts et al. [13] then re-utilized by Van Slambrouck et al. [14]. The approximate $h_{i}$ is defined as $h_{i}^{\delta}(x)=g_{i} \log (x)-x$ for $x \geq \delta$ and $h_{i}^{\delta}(x)=-\frac{\left(x-g_{i}\right)^{2}}{2 \delta}+g_{i} \log (\delta)-\delta+\frac{\left(\delta-g_{i}\right)^{2}}{2 \delta}$ for $x<\delta$. A similar framework can then be achieved by maximizing each $L^{\delta}+U$ as $\delta \rightarrow 0$, with $L^{\delta}(\boldsymbol{f}) \triangleq \sum_{i} h_{i}^{\delta}\left(\bar{g}_{i}(\boldsymbol{f})\right)$.

2) Solving the Sub-Problem: Solving (8) reduces to maximizing the unconstrained problems

$$
\text { maximize } L^{k}(\boldsymbol{f})+U(\boldsymbol{f}), \quad \boldsymbol{f} \in \mathbb{R}^{n_{\mathrm{v}}},
$$

for a sequence of $k$ 's. This can be achieved with any unconstrained optimization algorithm.

In this work, we utilized a limited-memory Broyden-FletcherGoldfarb-Shanno (L-BFGS) algorithm [23] to solve (11), for which we give a brief description. Assume we are estimating $\boldsymbol{f}^{k}$ from $\boldsymbol{f}^{k-1}$. Let $\Phi^{k} \triangleq L^{k}+U$ be the approximated PLL at iteration $k$ we wish to maximize. We define the first estimate as $\boldsymbol{f}^{k,(0)}=\boldsymbol{f}^{k-1}$. At inner-iteration $q$, given a current estimate $\boldsymbol{f}^{k,(q)}$, the new estimate $\boldsymbol{f}^{k,(q+1)}$ is obtained as

$$
\begin{aligned}
\boldsymbol{f}^{k,(q+1)} & =\boldsymbol{f}^{k,(q)}-s^{\star} \boldsymbol{B}^{k,(q)} \nabla \Phi^{k}\left(\boldsymbol{f}^{k,(q)}\right) \\
\text { with } s^{\star} & =\underset{s \in[0,1]}{\arg \max } \eta(s) \\
\text { and } \eta(s) & \triangleq \Phi^{k}\left(\boldsymbol{f}^{k,(q)}-s \boldsymbol{B}^{k,(q)} \nabla \Phi^{k}\left(\boldsymbol{f}^{k,(q)}\right)\right),
\end{aligned}
$$

where $\boldsymbol{B}^{k,(q)}$ is an approximate inverse Hessian of $\Phi^{k}$ evaluated at $\boldsymbol{f}^{k,(q)}$. The matrix/vector product $\boldsymbol{B}^{k,(q)} \nabla \Phi^{k}\left(\boldsymbol{f}^{k,(q)}\right)$ in
(12) is directly computed (without storing $\boldsymbol{B}^{k,(q)}$ ) from the $m$ previous iterates $\boldsymbol{f}^{k,(q-p)}, p=0, \ldots, m-1$.

For this paper we utilized the implementation from [24], which we also used in previous work [20]. Full details on the derivation of $\boldsymbol{B}^{k,(q)} \nabla \Phi^{k}\left(\boldsymbol{f}^{k,(q)}\right)$ can be found in [23], Chapter 6. The L-BFGS algorithm seeks for an approximate solution of (13). Our implementation used an iterative "backtracking" algorithm, consisting in gradually decreasing $s$ from $s^{\text {init }}=1$ until it satisfies the Wolfe Conditions [23], i.e.,

$$
\begin{aligned}
\eta\left(s^{\star}\right) & \geq \eta(0)+c_{1} s^{\star} \eta^{\prime}(0) \\
\eta^{\prime}\left(s^{\star}\right) & \leq c_{2} \eta^{\prime}(0)
\end{aligned}
$$

with $0<c_{1}<c_{2}<1$ (we used $c_{1}=10^{-4}$ and $c_{2}=0.9$ ). These conditions ensure a sufficient increase of $\eta(s)$ (condition (14)) as well as a sufficient decrease of the slope $\eta^{\prime}(s)$ (condition (15)). Furthermore, it can be demonstrated that if $s^{\star}$ satisfies both (14) and (15) then the next L-BFGS inverse Hessian approximate $\boldsymbol{B}^{k,(q+1)}$ is negative-definite (see [23], page 138), thus guaranteeing an ascent direction. The iterative scheme (w.r.t. $q$ ) is repeated until either the convergence criterion $\left\|\boldsymbol{f}^{k,(q+1)}-\boldsymbol{f}^{k,(q)}\right\| / \max \left\{\left\|\boldsymbol{f}^{k,(q+1)}\right\|,\left\|\boldsymbol{f}^{k,(q)}\right\|, 1\right\} \leq \tau$ is met or $q$ reaches a preset maximum number $N_{\text {inner }}$ of iterations.

The L-BFGS algorithm needs to be able to evaluate $\Phi^{k}$ and to compute $\nabla \Phi^{k}$, which is obtained by applying the chain rule on $L$ and $\varphi_{k}$. Each inner iteration $q$ requires a minimum of 2 projections/backprojections. As a surrogate of CPU time, we counted the number of projections/backprojections (including during the backtracking algorithm to find $s^{\star}$ in (13)), to compare the algorithms performance in Section III.

3) Algorithm Summary: Our methodology, namely hypoconvergence for penalized maximum-likelihood (HypoC-PML), is summarized in Algorithm 1. Any sequence $\left(\alpha_{k}\right)_{k}$ and $\left(\beta_{k}\right)_{k}$ such that $\alpha_{k} \beta_{k} \rightarrow+\infty$ can be used. We primarily used $\alpha_{k}=k^{2}$ and $\beta_{k}=k^{-1}$ but other sequences were considered (see Section III-C). Operation Define on lines 6, 9, 11 and 13 designates the creation of a mapping whereas Solve(problem; $\boldsymbol{x}, N)$ designates the output of an optimization algorithm applied to some optimization problem, initialized with a vector $\boldsymbol{x}$ and using a given solver (here L-BFGS) with $N$ iterations.

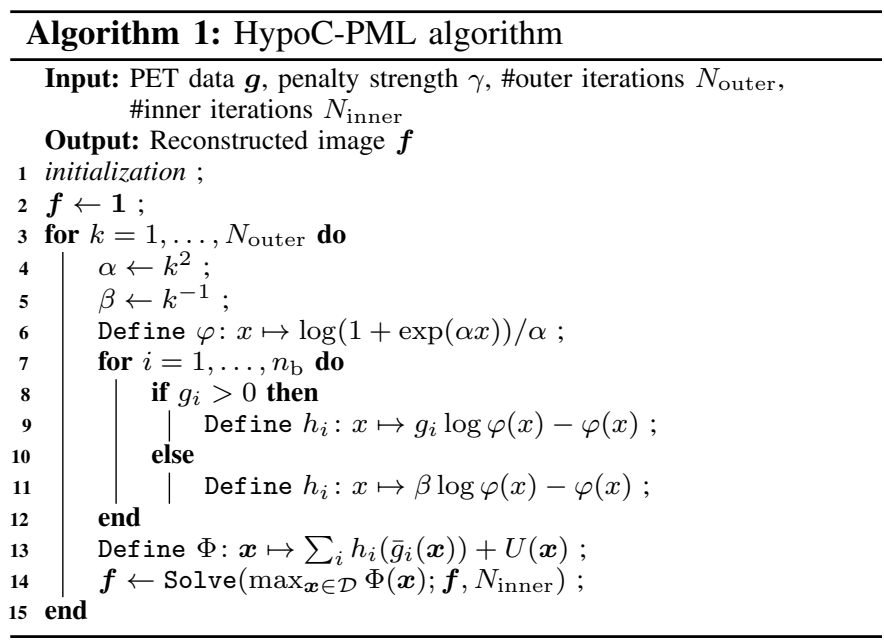




\section{Proof of Theorem 1}

We proceed to prove Theorem 1 by verifying below all the conditions required in Lemma A.1: Theorem 2 will check the conditions for $L^{k}+U$ and $L+U$ to have unique maximizers; Theorem 3 verifies the condition for the boundedness of the sequence $\left(\boldsymbol{f}^{k}\right)_{k}$; Theorem 3 and Remark 2 will imply the hypo-convergence of $L^{k}+U$ to $L+U$ (see Definition 1). Once all the conditions are satisfied, Theorem A.1 and Lemma A.1 readily imply that $\boldsymbol{f}^{k} \rightarrow \boldsymbol{f}^{*}$.

Theorem 2. The functions $L+U$ and $L^{k}+U$ (see (3), (6) and (9)), are proper and strictly concave, upper semi-continuous and coercive (see Definition A.1).

Proof: It is clear that $L$ and $L^{k}$ are proper and upper semi-continuous, while $U$ is continuous. It is also clear that $L$ and $L^{k}$ are concave functions (see lemma B.1) and, using the condition $\boldsymbol{g}^{\top} \boldsymbol{H} \mathbf{1}>0$ as in [7], it follows that $L+U$ and $L^{k}+U$ are strictly concave.

In the following the iteration number (superscript) $k$ is fixed, and to prove coercivity we introduce the sequence subscript $n$ and an arbitrary sequence $\left(\boldsymbol{f}_{n}\right)_{n}$ with $\left\|\boldsymbol{f}_{n}\right\| \rightarrow+\infty$. We want to prove that $L^{k}\left(\boldsymbol{f}_{n}\right)+U\left(\boldsymbol{f}_{n}\right) \rightarrow-\infty$ and $L\left(\boldsymbol{f}_{n}\right)+U\left(\boldsymbol{f}_{n}\right) \rightarrow$ $-\infty$. This is straightforward if $U\left(f_{n}\right) \rightarrow-\infty$, hence we assume $U\left(\boldsymbol{f}_{n}\right) \nrightarrow-\infty$. In such case there exists a sub-sequence $\left(n_{p}\right)_{p}$ and a voxel $j$ such that $\left[\boldsymbol{f}_{n_{p}}\right]_{j} \rightarrow \pm \infty$, let us say $+\infty$, as $p \rightarrow+\infty$ (the case $-\infty$ is similar), and $\left(U\left(\boldsymbol{f}_{n_{p}}\right)\right)_{p}$ is bounded. The neighboring system $j \mapsto \mathcal{N}_{j}$ being connected (c.f. the definition of $U$ ), we have $\left[\boldsymbol{f}_{n_{p}}\right]_{j} \rightarrow+\infty$ for all $j\left(\left(U\left(\boldsymbol{f}_{n_{p}}\right)\right)_{p}\right.$ is bounded, so if $\left[\boldsymbol{f}_{n_{p}}\right]_{j} \rightarrow+\infty$, then $\left[\boldsymbol{f}_{n_{p}}\right]_{j^{\prime}} \rightarrow+\infty$ for all $j^{\prime} \in \mathcal{N}_{j}$, and so on), so that $\left[\boldsymbol{H} \boldsymbol{f}_{n_{p}}\right]_{i} \rightarrow+\infty$ for at least one $i$, and $L^{k}\left(\boldsymbol{f}_{n_{p}}\right) \rightarrow-\infty$ and $L\left(\boldsymbol{f}_{n_{p}}\right) \rightarrow-\infty$ as $p \rightarrow+\infty$. The same rationale applies for $\left[\boldsymbol{f}_{n_{p}}\right]_{j} \rightarrow-\infty$. Since the sequence $\left(\boldsymbol{f}_{n}\right)_{n}$ is arbitrary, we have demonstrated that $L^{k}(\boldsymbol{f})+U(\boldsymbol{f}) \rightarrow$ $-\infty$ and $L(\boldsymbol{f})+U(\boldsymbol{f}) \rightarrow-\infty$ as $\|\boldsymbol{f}\| \rightarrow+\infty$.

Theorem 3. The sequence $\left(L^{k}\right)_{k}$ satisfies that $L^{k} \stackrel{h}{\rightarrow} L$ and there exists $G: \mathbb{R}^{n_{\mathrm{v}}} \rightarrow \mathbb{R}$ such that $G+U$ is coercive and $L^{k}(\boldsymbol{f}) \leq G(\boldsymbol{f})$ for all $\boldsymbol{f} \in \mathbb{R}^{n_{v}}$.

Proof: To prove that $L^{k} \stackrel{h}{\rightarrow} L$ we need to demonstrate that $L$ and $L^{k}$ satisfy (i) and (ii) of Definition 1.

Property (i) is clear since $\lim \sup _{k} L^{k}\left(f^{k}\right)=$ $\lim \sup _{k} \sum_{i} h_{i}^{k}\left(\bar{g}_{i}\left(\boldsymbol{f}^{k}\right)\right) \leq \sum_{i} \lim \sup _{k} h_{i}^{k}\left(\bar{g}_{i}\left(\boldsymbol{f}^{k}\right)\right)$ and Lemma B.1, parts b) and c), establishes that $h_{i}^{k} \stackrel{h}{\longrightarrow} h_{i}$. Hence, if $\boldsymbol{f}^{k} \rightarrow \boldsymbol{f}$, then $\limsup \sup _{k} L^{k}\left(\boldsymbol{f}^{k}\right) \leq L(\boldsymbol{f})$.

For property (ii), we observe that $\liminf _{k} L^{k}\left(f^{k}\right)=$ $\liminf _{k} \sum_{i} h_{i}^{k}\left(\bar{g}_{i}\left(\boldsymbol{f}^{k}\right)\right) \geq \sum_{i} \liminf _{k} h_{i}^{k}\left(\bar{g}_{i}\left(\boldsymbol{f}^{k}\right)\right)$. By the assumptions on $\boldsymbol{H}$ we have $\eta \triangleq \min _{i}\left(\max _{j}\left([\boldsymbol{H}]_{i, j}\right)\right)>0$, and if $\boldsymbol{f} \in \mathbb{R}^{n_{v}}$ then $\boldsymbol{f}^{k} \triangleq \boldsymbol{f}+\frac{\beta_{k}}{\eta} \mathbf{1}$ satisfies $\bar{g}_{i}\left(\boldsymbol{f}^{k}\right) \geq$ $\bar{g}_{i}(\boldsymbol{f})+\beta_{k}, \forall i$. Then Lemma B.1, parts b) and c), implies for this $\left(\boldsymbol{f}^{k}\right)_{k}$ that $\liminf _{k} h_{i}^{k}\left(\boldsymbol{f}^{k}\right) \geq h_{i}\left(\bar{g}_{i}(\boldsymbol{f})\right), \forall i$, hence $\liminf { }_{k} L^{k}\left(\boldsymbol{f}^{k}\right) \geq L(\boldsymbol{f})$.

We now prove the second part by letting $G(\boldsymbol{f}) \triangleq$ $\sum_{i=1}^{n_{b}} h_{i}^{0}\left(\bar{g}_{i}(\boldsymbol{f})\right)$. Using Lemma B.1, part a), we have that $G(\boldsymbol{f}) \geq L^{k}(\boldsymbol{f}), \forall k$. And using an argument similar to that of the proof of Theorem 2, it follows that $G+U$ is coercive.

\section{Results on Simulated DATA}

The benefit of utilizing negative values in PET for bias reduction in low-activity regions has already been demonstrated in previous work [11]-[15]. Our methodology solves the same optimization problem as the ADMM method from Lim et al. [15] (i.e., solving (8)), and thus converges to the same unique PML solution with the same properties (such as bias and noise). Therefore the objective of our simulations is to demonstrate that HypoC-PML achieves the desired results, which is the maximization of the PLL over the domain $\mathcal{D}$, using a simple and easy to implement approach, and compare its convergence speed with ADMM. It is expected that HypoCPML behaves similarly to standard PML algorithms over highactivity regions, but it should return a different reconstruction, possibly including negative values, over low-activity regions. In addition, HypoC-PML images are expected to be noisier over low-activity regions (such as the lungs) due to the absence of a lower-bound.

\section{A. General Settings}

All experiments were performed with $133 \times 133 \times 42$ volumes, with $3.125-\mathrm{mm}$ voxel-size. The projector $\boldsymbol{H}$ models a $5-\mathrm{mm}$ full-width at half-maximum (FWHM) PET system with 210 angles of view (parallel projections) and includes attenuation factors (phantom-dependent) as well as the acquisition time. The measurements were generated from a ground truth activity $f^{\text {truth }} \triangleq\left[f_{1}^{\text {truth }}, \ldots, f_{n_{\mathrm{v}}}^{\text {truth }}\right]^{\top} \in \mathbb{R}_{+}^{n_{\mathrm{v}}}$ following the Poisson model (1). The projected data comprised $33 \%$ and $66 \%$ of background events (corresponding to realistic randoms+scatter proportions in ${ }^{18} \mathrm{~F}$-fludeoxyglucose $\left({ }^{18} \mathrm{~F}\right.$-FDG) PET, see for example [25]), in the form of a uniform vector $\boldsymbol{r}$.

Activity images were reconstructed with M-MLEM, i.e., by solving the optimization problem (5) with positivity constraint on the image, using the optimization-transfer algorithm proposed in [5] and 400 iterations, and by solving the optimization problem (8) with both HypoC-PML and ADMM. For each method we used the same quadratic penalty term $U$ (see equation (6)) with $\psi(x)=\frac{1}{2} x^{2}$ and the standard 26 neighbors structure. Each methods used the same penalty weight $\gamma$. HypoC-PML was executed with $\left(\alpha_{k}, \beta_{k}\right)=\left(k^{2}, 1 / k\right)$, $\left(k^{2}, 1 / \log (k+1)\right)$ and $\left(k^{3}, k^{-1 / 2}\right)$, with $N_{\text {inner }}=70$ (maximum number of L-BFGS iterations on $q$ ) and $N_{\text {outer }}=25$. The corresponding reconstruction methods were denoted HypoCPML-1, HypoC-PML-2 and HypoC-PML-3. ADMM was implemented as described in Section III-B.

We proceeded with 2 experiments: with a cylindrical phantom (Section III-C) and with the extended cardiac-torso (XCAT) phantom [26] (Section III-D). The aim of the first experiment was to analyze the behavior of each method. The second experiment focused on a specific clinical application and only M-MLEM and HypoC-PML were considered.

\section{B. ADMM Implementation}

We implemented the ADMM methodology proposed by Lim et al. [15] to solve (8). At iteration $k$, the activity image estimate $\boldsymbol{f}^{k}$ is computed as

$$
\boldsymbol{f}^{k}=\underset{\boldsymbol{f} \in \mathbb{R}^{n_{\mathrm{v}}}}{\arg \min } \frac{\rho}{2}\left\|\boldsymbol{H} \boldsymbol{f}-\boldsymbol{v}^{k-1}+\boldsymbol{u}^{k-1}\right\|^{2}-U(\boldsymbol{f})
$$


whilst the auxiliary variable $\boldsymbol{v}$ and dual variable $\boldsymbol{u}$ are updated as

$$
\begin{aligned}
\boldsymbol{v}^{k} & =\underset{\boldsymbol{v} \geq-\boldsymbol{r}}{\arg \max } \sum_{i=1}^{n_{\mathrm{b}}} h_{i}\left(v_{i}+r_{i}\right)-\frac{\rho}{2}\left\|\boldsymbol{H} \boldsymbol{f}^{k}-\boldsymbol{v}+\boldsymbol{u}^{k-1}\right\|^{2} \\
\boldsymbol{u}^{k} & =\boldsymbol{u}^{k-1}+\boldsymbol{H} \boldsymbol{f}^{k}-\boldsymbol{v}^{k}
\end{aligned}
$$

While (17) can be updated analytically by zeroing the derivatives w.r.t. each $v_{i}$, the update (16) was computed iteratively. We utilized the L-BFGS algorithm-initialized with $f^{k-1}$ - on $\Psi^{k}(\boldsymbol{f}) \triangleq \frac{\rho}{2}\left\|\boldsymbol{H} \boldsymbol{f}-\boldsymbol{v}^{k-1}+\boldsymbol{u}^{k-1}\right\|^{2}-U(\boldsymbol{f})$ (c.f. Section II-C2).

The parameter $\rho$ is a penalty weight that tunes the proximity between $\boldsymbol{v}$ and $\boldsymbol{H} \boldsymbol{f}$. Its value does not affect the final result but it should be appropriately updated at each iteration $k$ for faster convergence. We proceeded as in [15] by using the parameter selection approach proposed by Boyd et al. [16]:

$$
\rho^{k+1}= \begin{cases}\tau^{\text {incr }} \rho^{k} & \text { if }\left\|\boldsymbol{a}^{k}\right\|>\mu\left\|\boldsymbol{b}^{k}\right\|, \\ \rho^{k} / \tau^{\text {decr }} & \text { if }\left\|\boldsymbol{b}^{k}\right\|>\mu\left\|\boldsymbol{a}^{k}\right\|, \\ \rho^{k} & \text { otherwise, }\end{cases}
$$

with $\boldsymbol{a}^{k}=\boldsymbol{H} \boldsymbol{f}^{k}-\boldsymbol{v}^{k}, \boldsymbol{b}^{k}=-\rho \boldsymbol{H}^{\top}\left[\boldsymbol{v}^{k}-\boldsymbol{v}^{k-1}\right], \tau^{\text {incr }}=$ $\tau^{\text {decr }}=2$ and $\mu=10$.

The utilization of $\boldsymbol{H}$ and $\boldsymbol{H}^{\top}$ in (17), (18) and (19) were taken into account in the CPU time (see Section II-C2). More specifically, the same projection $\boldsymbol{H} \boldsymbol{f}^{k}$ was used to compute $\boldsymbol{u}^{k}, \boldsymbol{v}^{k}$ and $\boldsymbol{a}^{k}$ while a separate backprojection was used for $b^{k}$.

We implemented 4 ADMM algorithms: (i) with a fixed value $\rho=1, N_{\text {inner }}=60$ and $N_{\text {outer }}=600$ (in order to be close to the optimum), denoted ADMM- $\rho_{\text {fixed }}$, and (ii) with an adaptive $\rho$, with $N_{\text {inner }}=5\left(N_{\text {outer }}=360\right), N_{\text {inner }}=30\left(N_{\text {outer }}=\right.$ $60)$ and $N_{\text {inner }}=90\left(N_{\text {outer }}=20\right)$, respectively denoted ADMM- $\rho_{\text {adapt }}-5$, ADMM- $\rho_{\text {adapt }}-30$ and ADMM- $\rho_{\text {adapt }}-90$. These algorithms, as well as HypoC-PML, converge to the same unique solution of (8).

\section{Experiment 1: Cylindrical Phantom}

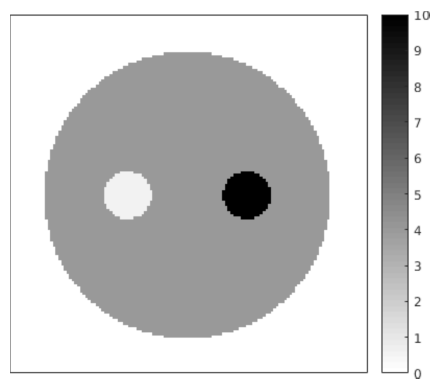

(a) Activity phantom

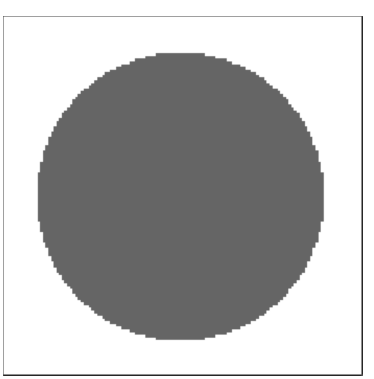

(b) Attenuation phantom
Fig. 1. Experiment 1-Activity and attenuation phantoms.

1) Simulation: We utilized a digital phantom (see Fig. 1) consisting of a $26-\mathrm{cm}$ diameter cylinder $\Omega$ containing two smaller cylinders $\Omega_{1}$ and $\Omega_{2}$. While $\Omega_{1}$ has a low-activity level (equals to 0.5 ), $\Omega_{2}$ has a high-activity level (equals to 10 ), and the activity level of the remaining region $\Omega \backslash\left(\Omega_{1} \cup \Omega_{2}\right)$ is low (equals to 4 ). The total number of counts was $11 \times 10^{6}$ (including background events) and we used $\gamma=5 \times 10^{-4}$ first then $\gamma=5 \times 10^{-3}$, which correspond to low- and highsmoothing. The attenuation in all of $\Omega$ was approximately the attenuation of water.

\section{2) Results:}

a) Reconstructed Images: Reconstructed images obtained from M-MLEM, ADMM- $\rho_{\text {fixed }}$ and HypoC-PML-1 are shown in Fig. 2. Reconstructions are comparable to the naked eye over hot regions, but differ on cold regions (cold spot and background) where the ADMM- $\rho_{\text {fixed }}$ and HypoC-PML-1 image values can be negative, also resulting in more noise in cold areas as expected. The same observation can be made when analyzing the reconstructed profiles in Fig. 3. We observe that HypoC-PML (1, 2 and 3$)$ and ADMM- $\rho_{\text {fixed }}$ reconstructions are identical, and coincide with M-MLEM over hot regions. With $\gamma=5 \times 10^{-4}$, HypoC-PML and ADMM$\rho_{\text {fixed }}$ reconstruct lower values (even negative) in the cold spot values to compensate for the spill-over from the surrounding activity. This phenomenon recedes with $\gamma=5 \times 10^{-3}$.

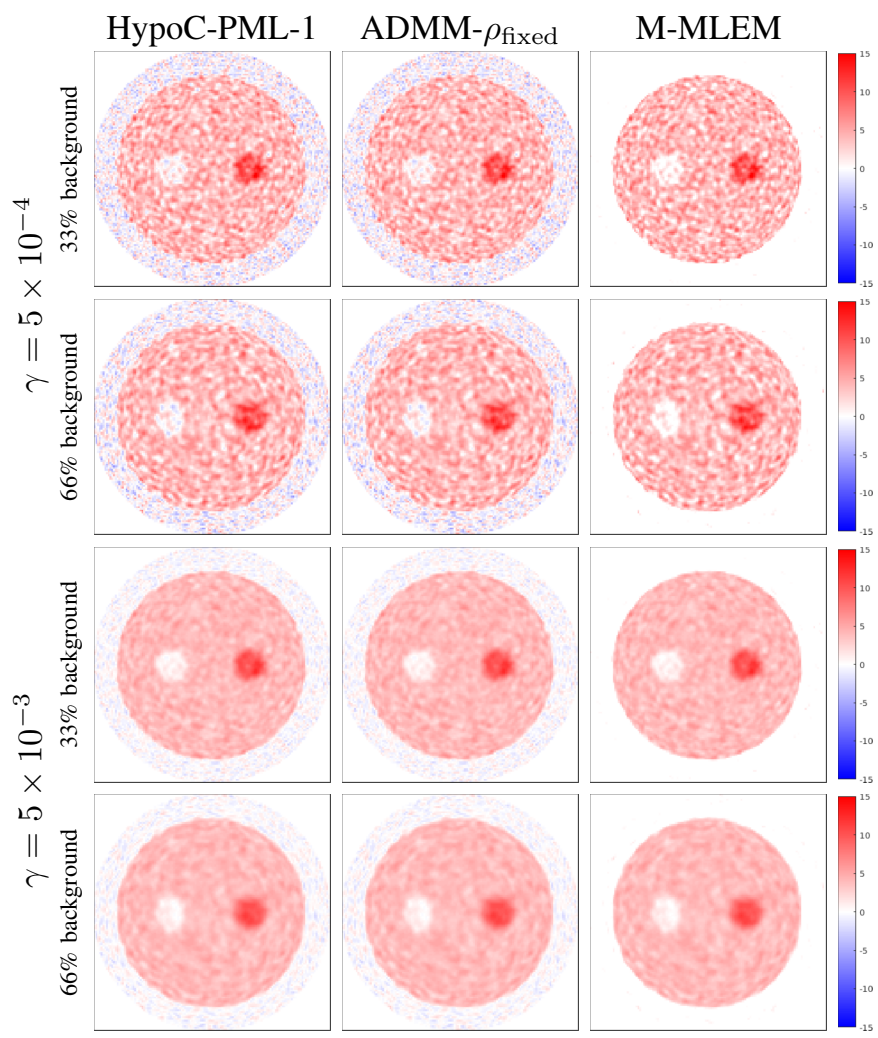

Fig. 2. Experiment 1-Phantom activity images reconstructed from simulated data comprising $33 \%$ and $66 \%$ of background events, using M-MLEM, ADMM$\rho_{\text {fixed }}$ and HypoC-PML-1, with $\gamma=5 \times 10^{-4}$ and $\gamma=5 \times 10^{-3}$.

The mean values are reported in Table I. The mean activity in the cold spot is over-estimated by each method (with a more severe bias with $66 \%$ background events). For $\gamma=5 \times 10^{-4}$ we observe that the bias reduced with ADMM- $\rho_{\text {fixed }}$ and HypoCPML, as compared with M-MLEM. However, the mean values are similar in the cold spot when using $\gamma=5 \times 10^{-3}$, suggesting that a strong penalty brings the solutions closer. The mean activity values in the hot spot are similar, with perhaps slightly higher values with M-MLEM. 
TABLE I

EXPERIMENT 1 -CYLINDRICAL PHANTOM MEAN ACTIVITY VALUES IN THE COLD AND HOT SPOTS.

\begin{tabular}{|c|c|c|c|c|c|c|c|c|}
\hline & \multicolumn{4}{|c|}{ Cold spot } & \multicolumn{4}{|c|}{ Hot spot } \\
\hline background & \multicolumn{2}{|c|}{$33 \%$} & \multicolumn{2}{|c|}{$66 \%$} & \multicolumn{2}{|c|}{$33 \%$} & \multicolumn{2}{|c|}{$66 \%$} \\
\hline$\gamma$ & $5 \times 10^{-4}$ & $5 \times 10^{-3}$ & $5 \times 10^{-4}$ & $5 \times 10^{-3}$ & $5 \times 10^{-4}$ & $5 \times 10^{-3}$ & $5 \times 10^{-4}$ & $5 \times 10^{-3}$ \\
\hline HypoC-PML-1 & 0.76198 & 0.92664 & 0.89122 & 1.1128 & 9.5015 & 9.1763 & 9.3541 & 8.838 \\
\hline HypoC-PML-2 & 0.75992 & 0.92652 & 0.88364 & 1.1198 & 9.5045 & 9.1762 & 9.3526 & 8.8411 \\
\hline HypoC-PML-3 & 0.75935 & 0.9213 & 0.88405 & 1.1126 & 9.5055 & 9.1868 & 9.3526 & 8.8472 \\
\hline ADMM- $\rho_{\text {fixed }}$ & 0.75973 & 0.92246 & 0.879 & 1.1191 & 9.5048 & 9.1831 & 9.3603 & 8.8402 \\
\hline M-MLEM & 0.86579 & 0.92013 & 1.0108 & 1.1162 & 9.5167 & 9.1951 & 9.3724 & 8.8544 \\
\hline true value & 0.5 & 0.5 & 0.5 & 0.5 & 10 & 10 & 10 & 10 \\
\hline
\end{tabular}

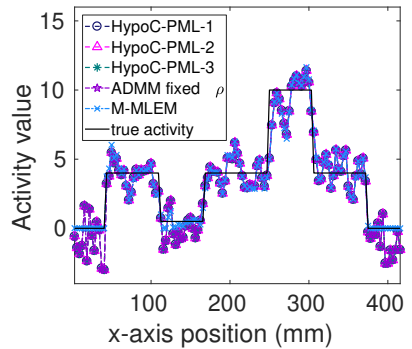

(a) $\gamma=5 \times 10^{-4}, 33 \%$ background

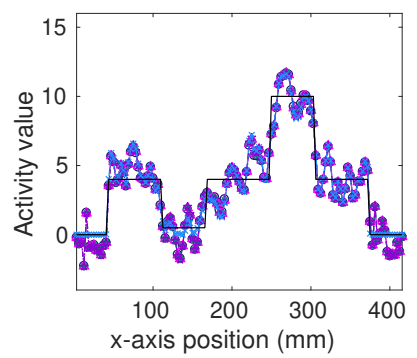

(c) $\gamma=5 \times 10^{-4}, 66 \%$ background

Fig. 3. Experiment $1-$ Reconstruction profiles of the cylindrical phantom.

Finally, Fig. 4 shows the simulated projection data $(66 \%$ background events) $\boldsymbol{g}$ at each bin $i$, as well the expected projection data $\overline{\boldsymbol{g}}(\boldsymbol{f})=\boldsymbol{H} \boldsymbol{f}+\boldsymbol{r}$ from M-MLEM, ADMM$\rho_{\text {fixed }}$ and HypoC-PML-1 $\left(\gamma=5 \times 10^{-4}\right)$, respectively denoted $\overline{\boldsymbol{g}}_{\mathrm{em}}, \overline{\boldsymbol{g}}_{\mathrm{admm}}$ and $\overline{\boldsymbol{g}}_{\mathrm{hc}}$, as well as the difference $\overline{\boldsymbol{g}}_{\mathrm{admm}}-\overline{\boldsymbol{g}}_{\mathrm{hc}}$. A similar figure was shown in [15]. It shows that $\overline{\boldsymbol{g}}_{\text {admm }}$ and $\overline{\boldsymbol{g}}_{\mathrm{hc}}$ appear identical and satisfy the new constraint $\boldsymbol{H} \boldsymbol{f}+\boldsymbol{r} \geq \mathbf{0}$, while $\overline{\boldsymbol{g}}_{\text {em }}$ is above $\boldsymbol{r}$ due to the positivity constraint on $\boldsymbol{f}$.

b) Convergence Speed: In order to investigate the convergence speed of each method, we calculated the normalized squared-error (NSE) between the current image estimate $f^{k}$ and the PML image $f^{\star}=\arg \max _{\mathcal{D}}(L+U)$ :

$$
\mathrm{NSE}=\frac{\left\|\boldsymbol{f}^{\star}-\boldsymbol{f}^{k}\right\|^{2}}{\left\|\boldsymbol{f}^{\star}\right\|^{2}}
$$

The PML image $f^{\star}$ is unique and can be obtained either from HypoC-PML or ADMM. We defined $f^{\star}$ as the image resulting from ADMM- $\rho_{\text {fixed }}$ in order to exclude convergence issues related to the parameter $\rho^{k}$ changing at each outer iteration $k$. We compared HypoC-PML-1, HypoC-PML-2 and HypoC-PML-3 with ADMM- $\rho_{\text {adapt }}-5$, ADMM- $\rho_{\text {adapt }}-30$ and ADMM- $\rho_{\text {adapt }}-90$ as well as with ADMM- $\rho_{\text {fixed }}$ and MMLEM, for $33 \%$ and $66 \%$ of background events and for $\gamma=5 \times 10^{-4}$ and $5 \times 10^{-3}$. Fig. 5 shows the NSE evaluated

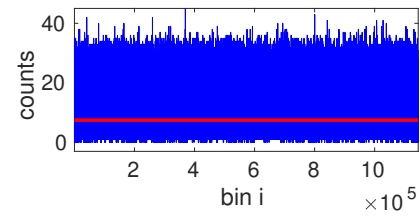

(a) Observed counts $\boldsymbol{g}$

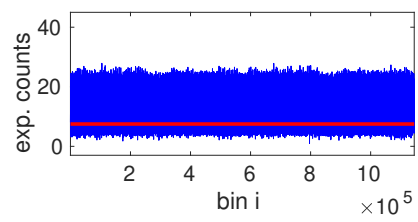

(c) Expected counts $\overline{\boldsymbol{g}}_{\text {admm }}$

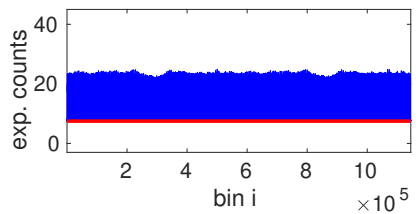

(b) Expected counts $\overline{\boldsymbol{g}}_{\text {em }}$

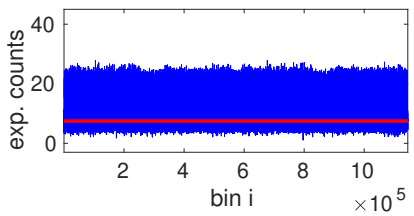

(d) Expected counts $\overline{\boldsymbol{g}}_{\mathrm{hc}}$

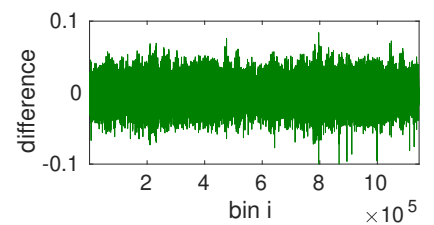

(e) Difference $\overline{\boldsymbol{g}}_{\text {admm }}-\overline{\boldsymbol{g}}_{\mathrm{hc}}$

Fig. 4. Experiment $1-$ (a) Simulated data $g$ (66\% background); (b)-(d) Expected sinograms $\boldsymbol{H} \boldsymbol{f}+\boldsymbol{r}$ resulting from M-MLEM, ADMM- $\rho_{\text {fixed }}$ and HypoC-PML-1 $\left(\overline{\boldsymbol{g}}_{\mathrm{em}}, \overline{\boldsymbol{g}}_{\text {admm }}\right.$ and $\left.\overline{\boldsymbol{g}}_{\text {hc }}\right)$ with $\gamma=5 \times 10^{-4}$ (the red line represents the expected background $\boldsymbol{r}$, while the blue curve represents the sinogram at each bin $i$ ) (e) Difference $\overline{\boldsymbol{g}}_{\mathrm{admm}}-\overline{\boldsymbol{g}}_{\mathrm{hc}}$. This figure was inspired from [15].

at each inner iteration $q$ and plotted against the total number of projections/backprojections, which represents the CPU time. For display purpose M-MLEM was ran with over 2,000 iterations.

Results show that the HypoC-PML methods behave similarly and achieve faster convergence than ADMM methods, regardless of the background and $\gamma$. When $\gamma=5 \times 10^{-3}$, ADMM$\rho_{\text {adapt }}-5$ at early iterations is comparable to HypoC-PML, but it is outperformed by ADMM- $\rho_{\text {adapt }}-30$ and HypoC-PML at later iterations. ADMM- $\rho_{\text {fixed }}$ has the slowest convergence rate while M-MLEM has the fastest (but converges to a different solution). While ADMM- $\rho_{\text {adapt }}$ methods achieve faster convergence than ADMM- $\rho_{\text {fixed }}$, their convergence is non-monotonic as $\rho$ is changed at each iteration.

\section{Experiment 2: XCAT Phantom}

1) Simulation: We utilized the XCAT activity and attenuation phantoms (Fig. 6). The phantom contains 2 tumors (hot lesions): a $3 \mathrm{~cm}$-diameter tumor in the liver and a $5 \mathrm{~cm}$ diameter tumor in the lungs, the latter containing a necrosis (no activity). We generated data with $N_{\text {count }}=20 \times 10^{6}$ 


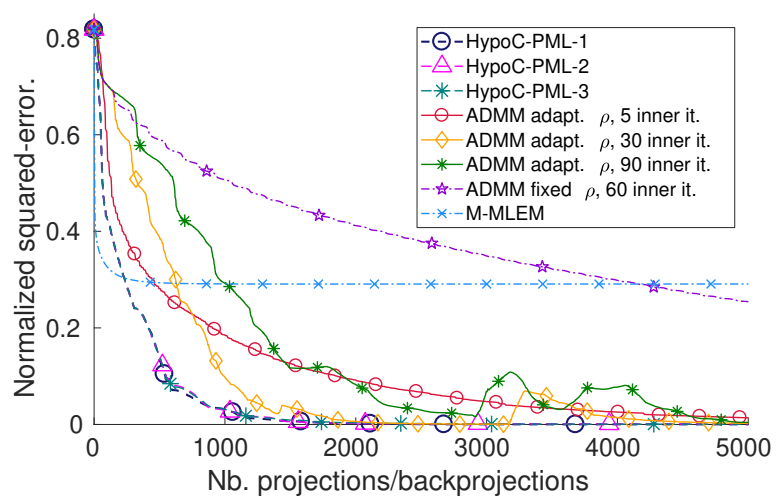

(a) $\gamma=5 \times 10^{-4}, 33 \%$ background

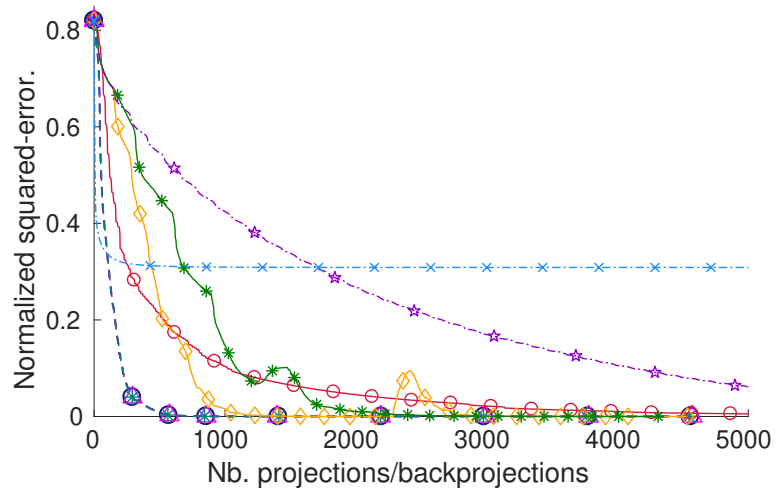

(c) $\gamma=5 \times 10^{-4}, 66 \%$ background

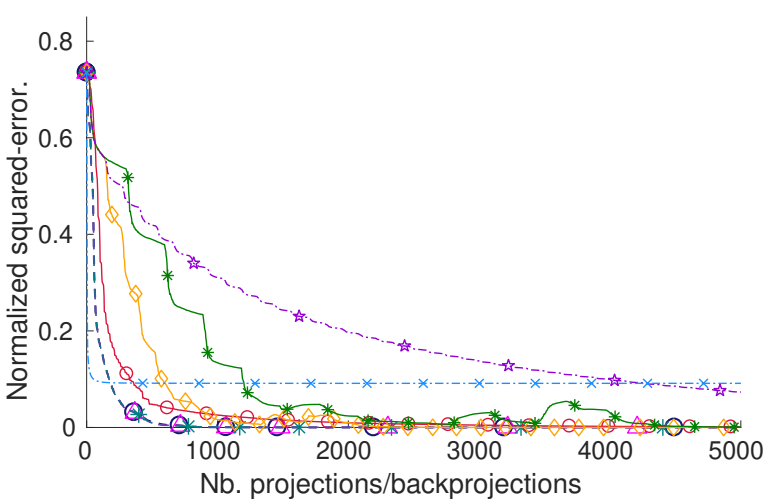

(b) $\gamma=5 \times 10^{-3}, 33 \%$ background

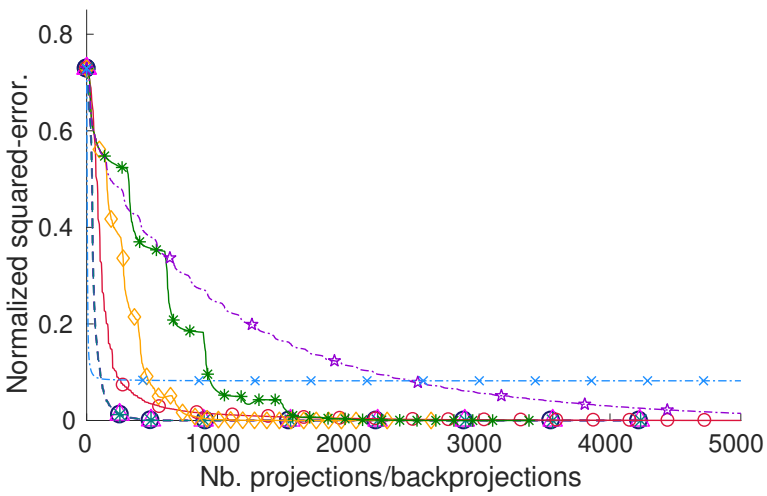

(d) $\gamma=5 \times 10^{-3}, 66 \%$ background

Fig. 5. Experiment 1 -NSE (as defined in (20)) plotted against the total number of projections/backprojections at each inner iteration $q$, for each of the considered methods, with $33 \%$ and $66 \%$ background events, and $\gamma=5 \times 10^{-3}$ and $\gamma=5 \times 10^{-4}$.

to $60 \times 10^{6}$ and $N_{\text {count }}=60 \times 10^{6}$ counts, with $33 \%$ and $66 \%$ background events. HypoC-PML was implemented with $\left(\alpha_{k}, \beta_{k}\right)=\left(k^{2}, 1 / k\right)$ (HypoC-PML-1). $N_{\text {noise }}=40$ independent noise realizations were simulated. The activity distribution was reconstructed using M-MLEM and HypoCPML, but this time we imposed the activity to be 0 in the background (outside of the patient). We considered 2 error functions: the mean (relative) bias

$$
\text { Bias }=\frac{1}{\# \mathcal{R}} \frac{1}{N_{\text {noise }}} \sum_{j \in \mathcal{R}} \sum_{\ell=1}^{N_{\text {noise }}} \hat{f}_{j}^{[\ell]}-f_{j}^{\text {truth }},
$$

and the mean absolute bias

$$
\operatorname{AbsBias}=\frac{1}{\# \mathcal{R}} \frac{1}{N_{\text {noise }}} \sum_{j \in \mathcal{R}} \sum_{\ell=1}^{N_{\text {noise }}}\left|\hat{f}_{j}^{[\ell]}-f_{j}^{\text {truth }}\right|
$$

where $\hat{f}_{j}^{[\ell]}$ denotes the reconstructed activity at voxel $j$ from the $\ell$-th noise replicate, $\mathcal{R}$ is a region of interest (e.g., a tumor) and $\# \mathcal{R}$ denotes the number of voxels in $\mathcal{R}$. We also used the mean standard deviation (STD),

$$
\mathrm{STD}=\frac{1}{\# \mathcal{R}} \sum_{j \in \mathcal{R}} \sqrt{\frac{1}{N_{\text {noise }}} \sum_{\ell=1}^{N_{\text {noise }}}\left(\hat{f}_{j}^{[\ell]}-\overline{\hat{f}}_{j}\right)^{2}},
$$

where $\overline{\hat{f}}_{j}^{[\ell]}=N_{\text {noise }}^{-1} \sum_{\ell=1}^{N_{\text {noise }}} \hat{f}_{j}^{[\ell]}$.

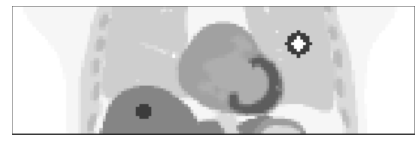

(a) Activity phantom

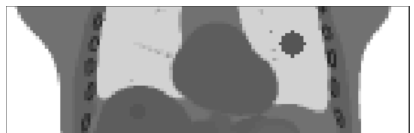

(b) Attenuation phantom
Fig. 6. Experiment 2-(a) Activity and (b) attenuation XCAT phantoms.

2) Results: M-MLEM and HypoC-PML reconstructed activity images are shown in Fig. 7. For each count level, we used $\gamma=\left(N_{\text {count }} / N_{\text {count }}^{\text {ref }}\right) \times \gamma^{\text {ref }}, \gamma^{\text {ref }}=4 \times 10^{-3}$ and $N_{\text {count }}^{\text {ref }}=60 \times 10^{6}$, in order to maintain the degree of smoothness. We used $\gamma^{\text {ref }}=4 \times 10^{-3}$ and $\gamma^{\text {ref }}=3 \times 10^{-3}$ for the $33 \%$ and $66 \%$ background events simulations respectively.

The images appear identical in hot regions (heart, liver and tumors) but the HypoC-PML images contain negative values in cold areas (the lungs and the tumor necrosis). The activity being lower in the lungs, HypoC-PML-reconstructed lung activity values are noisier than that of M-MLEM. This phenomenon is observed with any reconstruction method that allows for negative values in the activity image [11]-[15].

Fig. 8 shows the reconstruction profiles across the 2 lesions (foot to head) for M-MLEM and HypoC-PML with $60 \times 10^{6}$ counts. As observed in the images, the profiles appear similar in hot regions. However the HypoC-PML activity profiles show signs of variability in cold regions. In particular, the HypoC-PML activity in the necrosis appears significantly lower 


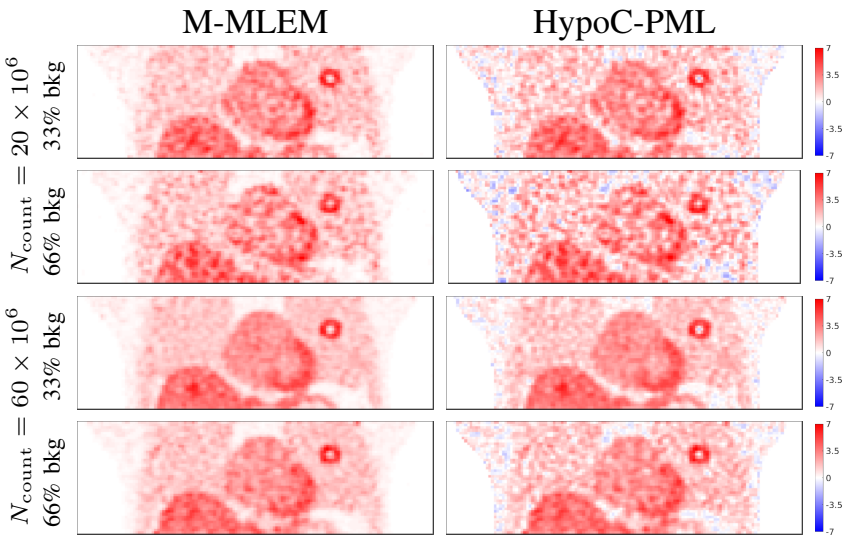

Fig. 7. Experiment 2-Reconstructed activity with $N_{\text {count }}=20 \times 10^{6}$ and $60 \times 10^{6}, 33 \%$ and $66 \%$ background events, using M-MLEM and HypoC-PML.

compared with M-MLEM, which confirms the observations made in Section III-C.
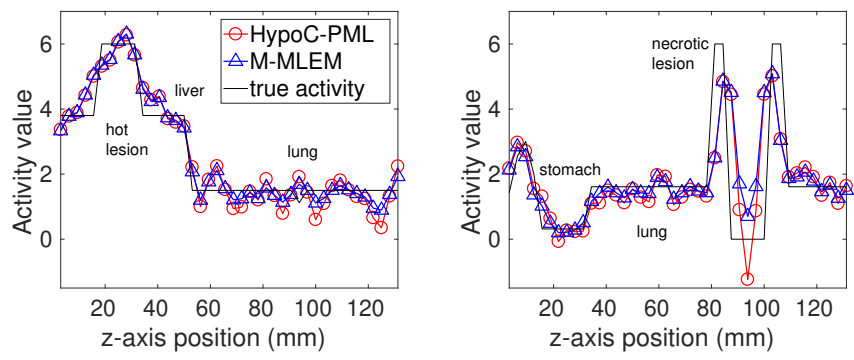

(a) Lesion 1 (liver), 33\% background

(b) Lesion 2 (necrosis), 33\% background

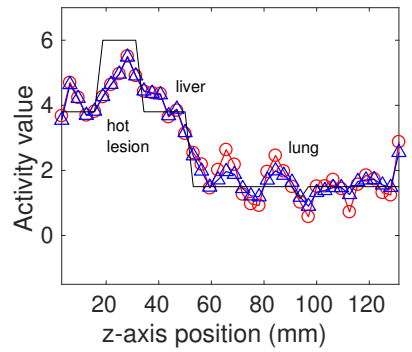

(c) Lesion 1 (liver), 66\% background

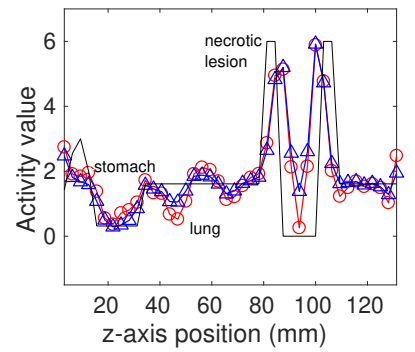

(d) Lesion 2 (necrosis), 66\% background

Fig. 8. Experiment 2-Reconstruction profiles across lesion 1 (liver) and lesion 2 (containing a necrosis), with $N_{\text {count }}=60 \times 10^{6}, 33 \%$ and $66 \%$ background events, using M-MLEM and HypoC-PML.

In order to assess the effect of $\gamma$ on quantification, we plotted the mean absolute bias (22) in lesion 1 and the mean bias (21) in the necrosis of lesion 2 against the STD (23) in a sub-region of the liver, for $N_{\text {count }}=20 \times 10^{6}$ then $N_{\text {count }}=60 \times 10^{6}$, with 7 values of $\gamma$ ranging from $\gamma=10^{-3}$ to $\gamma=5 \times 10^{-2}$ (for $N_{\text {count }}=20 \times 10^{6}$ ) then $\gamma=3 \times 10^{-3}$ to $\gamma=1.5 \times 10^{-1}$ for $\left(N_{\text {count }}=60 \times 10^{6}\right)$. The results are displayed in Fig. 9 and Fig. 10. While the bias/STD curves are comparable on lesion 1, we observe that the bias on the necrosis (lesion 2) using HypoC-PML is smaller than that of M-MLEM (for a similar noise level) for small $\gamma$-values. The curves coincide for large $\gamma$-values as the penalty is predominant in the cost function. This result indicates that allowing for negativity in the reconstructed image allows to reduce the bias in the necrosis while keeping the noise under control.

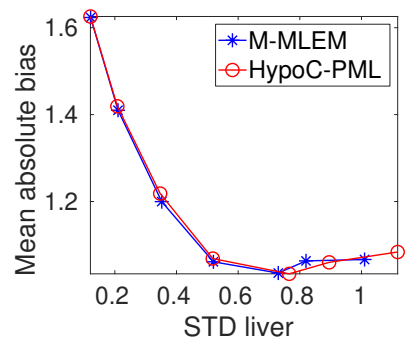

(a) Lesion 1,33\% background

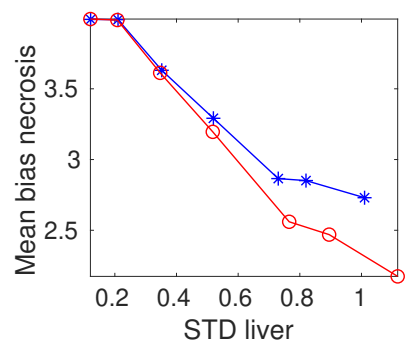

(c) Lesion 2, 33\% background

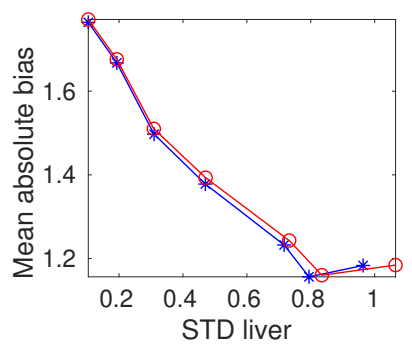

(b) Lesion 1, 66\% background

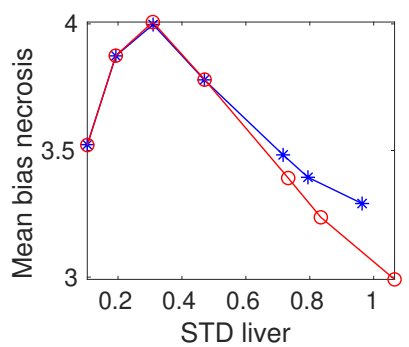

(d) Lesion 2, 66\% background
Fig. 9. Experiment 2-Mean bias (lesion 1) and mean absolute bias (necrosis in lesion 2) versus STD (sub-region of the liver) of the M-MLEM- and HypoCPML-reconstructed activity images from the $N_{\text {count }}=20 \times 10^{6}$ simulations, with $\gamma \in\left[10^{-3}, 5 \times 10^{-2}\right]$. Each point correspond to a single $\gamma$-value. The values were calculated following (21), (22) and (23), with $N_{\text {noise }}=40$ noise replicates. High STD points (right end of each curve) correspond to the low $\gamma$-values.

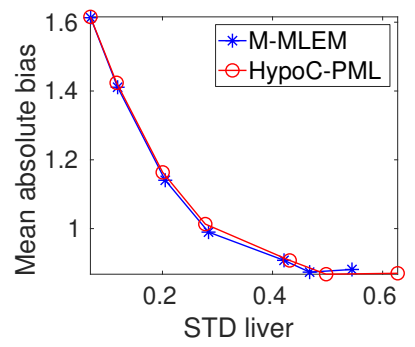

(a) Lesion 1,33\% background

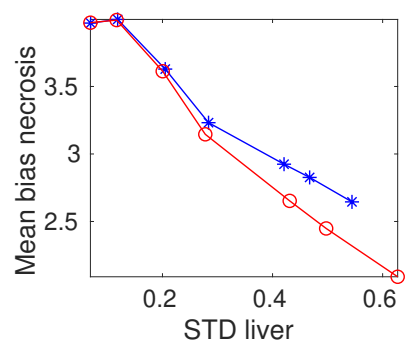

(c) Lesion 2, 33\% background

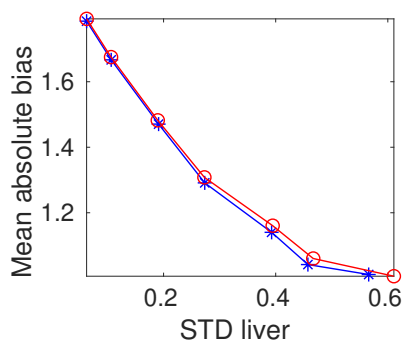

(b) Lesion $1,66 \%$ background

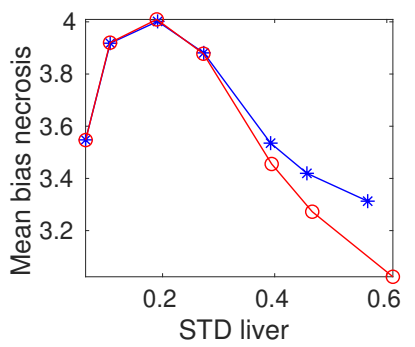

(d) Lesion 2, 66\% background
Fig. 10. Experiment 2-Same as Fig. 9 with $N_{\text {count }}=60 \times 10^{6}$ and $\gamma \in\left[3 \times 10^{-3}, 1.5 \times 10^{-1}\right]$.

\section{DISCUSSION}

Our hypo-convergence based algorithm converges to a solution of the PML problem (8), i.e., with positivity constraints in the projection space. M-MLEM and HypoC-PML 
reconstructions coincide on high-activity regions, where the positivity constraint is not active, and differ on low-activity regions, where HypoC-PML can reduce the bias by allowing for negative values.

In terms of reconstructed images, HypoC-PML provides the same results as ADMM as both approaches aim at solving the same problem (8), but HypoC-PML converges faster. The main difference resides in the methodology used to solve the problem. While ADMM elegantly transfers the constraints to the projections space only, its convergence speed is heavily affected by fine tuning of $\rho$, which might be challenging. On the other hand, HypoC-PML appears more practical, provided that a solver to maximize $L_{k}$ is available, and convergence is guaranteed. Besides, in the experiments presented here, HypoCPML appears to be robust with respect to the choice of the sequences $\left(\alpha_{k}\right)_{k}$ and $\left(\beta_{k}\right)_{k}$.

In principle, $\mathrm{ADMM}$ can be applied to impose the positivity constraint for any figure of merit other than the Poisson loglikelihood. Although HypoC-PML, as presented here, is tailored for the Poisson log-likelihood, the same methodology can be easily extended to other figures of merit with positivity constraints. For example, if $h_{i}$ in (3) is replaced by a continuous function $u_{i}: \mathbb{R} \rightarrow \mathbb{R}$ (e.g., for a weighted leastsquares cost), then the sequence $\left(u_{i}^{k}\right)_{k}$ defined for all $k$ as $u_{i}^{k}: x \mapsto u_{i}(x)+\beta_{k} \log \varphi_{k}(x)$ hypo-converges to $\tilde{u}_{i}: \mathbb{R} \rightarrow \overline{\mathbb{R}}$ with $\tilde{u}_{i}(x)=u_{i}(x)$ for $x \geq 0$ and $\tilde{u}_{i}(x)=-\infty$ for $x<0$ (under the same conditions prescribed for $\alpha_{k}, \beta_{k}$ ). Therefore, $\sum_{i} u_{i}\left(\bar{g}_{i}(\boldsymbol{x})\right)$ can be maximized with positivity constraint $\bar{g}_{i}(\boldsymbol{x}) \geq 0$ by sequentially maximizing $\sum_{i} u_{i}^{k}\left(\bar{g}_{i}(\boldsymbol{x})\right)$ without constraints, using the same methodology.

Having negative values in PET images has no real quantitative meaning at the voxel level, nevertheless the bias reduction in low activity regions is useful for specific tasks, such as region-based dynamic imaging and tumor texture analysis.

\section{CONCLUSION}

We proposed a new methodology, namely, HypoC-PML, for PML PET reconstruction, with positivity constraints on the projections as opposed to positivity constraints on the image values, which allows for some degree of negativity in the reconstructed image. Our method consists of maximizing each term of a sequence of intermediary objective functions that hypo-converges to the PLL. Each intermediary objective function can be maximized without constraints. We gave a mathematical proof that the resulting images sequence converges to the solution of the main problem. Results on simulated data (geometrical and XCAT phantoms) showed that HypoC-PML and ADMM deliver activity images with lower bias than M-MLEM in cold area, but HypoC-PML converges faster than ADMM. Further work includes applications to quantitative analysis such as dynamic imaging and tumor homogeneity analysis.

\section{APPENDIX A}

\section{HYPO-CONVERGENCE AND MAXIMIZERS}

The hypo-convergence is related to maximization problems through the following result:
Theorem A.1. Let $F, F_{k}: \mathbb{R}^{n} \rightarrow \overline{\mathbb{R}}, k \in \mathbb{N}$ be a collection of functions bounded from above. Assume that $F_{k} \stackrel{h}{\longrightarrow} F$, and assume $\left(\boldsymbol{x}_{k}^{\star}\right)_{k}$ is a sequence in $\mathbb{R}^{n}$ such that $\boldsymbol{x}_{k}^{\star} \in \arg \max \left(F_{k}\right)$ and $\boldsymbol{x}_{k}^{\star} \rightarrow \boldsymbol{x}^{\star}$. Then $\boldsymbol{x}^{\star} \in \arg \max (F)$.

Proof: In a more general setting this can be found in [27], we present a direct proof in this simplified setting. We first observe that, since $F^{k} \stackrel{h}{\longrightarrow} F$, we have

$$
F\left(\boldsymbol{x}^{\star}\right) \geq \limsup _{k} F_{k}\left(\boldsymbol{x}_{k}^{\star}\right) \geq \liminf _{k}\left(\sup _{\mathbb{R}^{n}}\left(F_{k}\right)\right) .
$$

Next, if $\sup _{\mathbb{R}^{n}}(F)<+\infty$, then for $\epsilon>0, \exists \boldsymbol{z} \in \mathbb{R}^{n}$ such that $F(\boldsymbol{z}) \geq \sup _{\mathbb{R}^{n}}(F)-\epsilon$. Since $F_{k} \stackrel{h}{\rightarrow} F$, there exists $\boldsymbol{z}_{k} \rightarrow \boldsymbol{z}$ such that $\liminf _{k} F_{k}\left(\boldsymbol{z}_{k}\right) \geq F(\boldsymbol{z})$ and

$$
\sup _{\mathbb{R}^{n}}(F)-\epsilon \leq F(\boldsymbol{z}) \leq \liminf _{k} F_{k}\left(\boldsymbol{z}_{k}\right) \leq \liminf _{k}\left(\sup _{\mathbb{R}^{n}}\left(F_{k}\right)\right) .
$$

Together, the above inequalities imply $F\left(\boldsymbol{x}^{\star}\right) \geq \sup _{\mathbb{R}^{n}}(F)-\epsilon$ for $\epsilon>0$ arbitrary, hence we conclude that $F\left(\boldsymbol{x}^{\star}\right) \geq \sup _{\mathbb{R}^{n}}(F)$ and $\boldsymbol{x}^{\star} \in \arg \max (F)$.

There are different settings under which hypo-convergence will furthermore imply that $\arg \max \left(F_{k}\right) \rightarrow \arg \max (F)$ in some sense. We detail one such setting that is not the most general, but that is simple and it will be useful enough for us.

Definition A.1. Let $F: \mathbb{R}^{n} \rightarrow \overline{\mathbb{R}}$.

a) We say that $F$ is proper and strictly concave if $F$ is not identically $-\infty$ and

$$
\begin{aligned}
& \forall \boldsymbol{x}, \boldsymbol{y} \in F^{-1}(\mathbb{R}), \forall \lambda \in[0,1] \\
& F(\lambda \boldsymbol{x}+(1-\lambda) \boldsymbol{y})>\lambda F(\boldsymbol{x})+(1-\lambda) F(\boldsymbol{y}) .
\end{aligned}
$$

b) We say that $F$ is coercive if $F(\boldsymbol{x}) \rightarrow-\infty$ as $\|\boldsymbol{x}\| \rightarrow+\infty$. c) We say that $F$ is upper semi-continuous if $\lim \sup _{k} F\left(\boldsymbol{x}_{k}\right) \leq F(\boldsymbol{x})$ for all $\boldsymbol{x}$ and for all $\boldsymbol{x}_{k} \rightarrow \boldsymbol{x}$.

Lemma A.1. Assume $F, F_{k}: \mathbb{R}^{n} \rightarrow \overline{\mathbb{R}}$ are proper and strictly concave, coercive and upper semi-continuous functions. Then,

a) Uniqueness of the maximizers: there exists $\boldsymbol{x}^{\star}, \boldsymbol{x}_{k}^{\star} \in$ $\mathbb{R}^{n}$ such that $\left\{\boldsymbol{x}^{\star}\right\}=\arg \max (F)$ and $\left\{\boldsymbol{x}_{k}^{\star}\right\}=$ $\arg \max \left(F_{k}\right), \forall k \in \mathbb{N}$.

b) Convergence of bounded approximates: if $F_{k} \stackrel{h}{\rightarrow} F$ and $\left(\boldsymbol{x}_{k}^{\star}\right)_{k}$ is a bounded sequence in $\mathbb{R}^{n}$, then $\boldsymbol{x}_{k}^{\star} \rightarrow \boldsymbol{x}^{\star}$.

c) Boundedness of the approximates: if $F_{k} \stackrel{h}{\rightarrow} F$ and there exists $G$ coercive function such that $G(\boldsymbol{x}) \geq F_{k}(\boldsymbol{x})$ for all $\boldsymbol{x} \in \mathbb{R}^{n}$ then $\left(\boldsymbol{x}_{k}^{\star}\right)_{k}$ is a bounded sequence in $\mathbb{R}^{n}$.

Proof: Part a) is a standard result (see [27]).

For part b), since $\arg \max (F)=\left\{\boldsymbol{x}^{\star}\right\}$, Theorem A.1 implies that any cluster point of the sequence $\left(\boldsymbol{x}_{k}^{\star}\right)_{k}$ has to be equal to $\boldsymbol{x}^{\star}$. But $\left(\boldsymbol{x}_{k}^{\star}\right)_{k} \subset \mathbb{R}^{n}$ is bounded and $\boldsymbol{x}^{\star}$ is the only possible cluster point of the sequence, hence $\boldsymbol{x}_{k}^{\star} \rightarrow \boldsymbol{x}^{\star}$.

For part c), fix $z \in F^{-1}(\mathbb{R})$ and $z_{k} \rightarrow z$ such that $\lim \inf _{k} F_{k}\left(\boldsymbol{z}_{k}\right) \geq F(\boldsymbol{z})$ (such a sequence exists due to hypo-convergence), hence $\exists K \in \mathbb{N}$ such that $F_{k}\left(\boldsymbol{z}_{k}\right) \geq$ $F(\boldsymbol{z})-1, \forall k \geq K$. Since $G$ is coercive, the set $B=\{\boldsymbol{x} \in$ $\left.\mathbb{R}^{n}: G(\boldsymbol{x}) \geq F(\boldsymbol{z})-1\right\}$ is bounded. Then, for $k \geq K$, $F(\boldsymbol{z})-1 \leq F_{k}\left(\boldsymbol{z}_{k}\right) \leq F_{k}\left(\boldsymbol{x}_{k}^{\star}\right) \leq G\left(\boldsymbol{x}_{k}^{\star}\right)$ and we conclude that $\left(\boldsymbol{x}_{k}^{\star}\right)_{k \geq K} \subset B$ and $\left(\boldsymbol{x}_{k}^{\star}\right)_{k}$ is bounded. 


\section{APPENDIX B \\ HyPo-CONVERGENCE AND EQUI-COERCIVITY OF THE ELEMENTARY FUNCTIONS}

In this section we demonstrate the hypo-convergence to $h_{i}$ (see (4)) of the sequence $\left(h_{i}^{k}\right)_{k}$ (see (10)) and its equi-coercivity. For this purpose, we define $\alpha_{0} \triangleq \min _{k}\left(\alpha_{k}\right), \beta_{0} \triangleq \max _{k}\left(\beta_{k}\right)$, and $\kappa_{0}=\min _{k}\left(\alpha_{k} \beta_{k}\right)$ and we will assume that $\alpha_{k} \beta_{k} \rightarrow+\infty$. Moreover, by moving forward in the sequences, we can assume without loss of generality that $\kappa_{0}>0, \alpha_{0} \geq 1$ and $\beta_{0} \leq \mathrm{e}$. We also define the functions $h_{i}^{0}: \mathbb{R} \rightarrow \mathbb{R}$ which will play the role of $G$ in Lemma A.1 for the collection $\left(h_{i}^{k}\right)_{k}$ as:

$h_{i}^{0}(x) \triangleq \begin{cases}g_{i} \log \varphi_{0}(x)-\max (0, x) & \text { if } g_{i}>0 \& x \in \mathbb{R}, \\ \beta_{0} \log \varphi_{0}(x)-x & \text { if } g_{i}=0 \& \varphi_{0}(x)>1, \\ \kappa_{0} x & \text { if } g_{i}=0 \& \varphi_{0}(x) \leq 1,\end{cases}$

where $\varphi_{0}$ follows the same definition as $\varphi_{k}$ below equation (10).

Lemma B.1. We have the following properties on $h_{i}^{k}$ :

a) For $x \in \mathbb{R}$ we have $\max (0, x)<\varphi_{k}(x) \leq \max (0, x)+$ $\log (2) / \alpha_{k}$. Also, for each $k \in \mathbb{N}, \varphi_{k}(x)$ is strictly increasing in $x$, and if $\alpha_{k}<\alpha_{\ell}$ then $\varphi_{k}(x)>\varphi_{\ell}(x), \forall x \in \mathbb{R}$ (i.e., strictly decreasing in $\alpha$ ).

b) If $g_{i}>0$, then $h_{i}^{k}: \mathbb{R} \rightarrow \mathbb{R}, k \in \mathbb{N}$, are strictly concave and continuous, and for all $x \in \mathbb{R}$ and all $x_{k} \rightarrow x$ we have $h_{i}^{k}\left(x_{k}\right) \rightarrow h_{i}(x)$.

c) If $g_{i}=0$, then $h_{i}^{k}(x) \triangleq \beta_{k} \log \varphi_{k}(x)-\varphi_{k}(x)$ are such that $h_{i}^{k}: \mathbb{R} \rightarrow \mathbb{R}$ are strictly concave and continuous. And for all $x \in \mathbb{R} \backslash\{0\}$ and all $x_{k} \rightarrow x$ we have $h_{i}^{k}\left(x_{k}\right) \rightarrow h_{i}(x)$, while for $x=0$ we have

(i) $\quad \limsup _{k} h_{i}^{k}\left(x_{k}\right) \leq h_{i}(0)$ for all $x_{k} \rightarrow 0$, and

(ii) $\quad \liminf _{k} h_{i}^{k}\left(x_{k}\right) \geq h_{i}(0)$ for all $x_{k} \rightarrow 0, x_{k} \geq \beta_{k}$.

Moreover, $h_{i}^{k} \leq h_{i}^{0}, \forall k \in \mathbb{N}$, and $h_{i}^{0}$ is coercive.

Proof:

a) For $x>0$ we have

$$
\begin{aligned}
\varphi_{k}(x)-x & =\frac{\log \left(1+\mathrm{e}^{\alpha_{k} x}\right)}{\alpha_{k}}-\frac{\log \left(\mathrm{e}^{\alpha_{k} x}\right)}{\alpha_{k}} \\
& \left.=\frac{1}{\alpha_{k}} \log \left(\mathrm{e}^{-\alpha_{k} x}+1\right) \in\right] 0, \log (2) / \alpha_{k}[
\end{aligned}
$$

For $x \leq 0$ we have $0<\mathrm{e}^{\alpha_{k} x} \leq 1$ and $\varphi_{k}(x)=\log (1+$ $\left.\left.\left.\mathrm{e}^{\alpha_{k} x}\right) / \alpha_{k} \in\right] 0, \log (2) / \alpha_{k}\right]$. In summary, for all $x \in \mathbb{R}$,

$$
0<\varphi_{k}(x)-\max (0, x) \leq \frac{\log (2)}{\alpha_{k}}
$$

Additionally, for $\alpha>0$ and $x \in \mathbb{R}$,

$$
\begin{aligned}
\frac{\partial}{\partial \alpha}\left(\frac{\log \left(1+\mathrm{e}^{\alpha x}\right)}{\alpha}\right) & =\frac{1}{\alpha^{2}}\left(\frac{\log \left(\mathrm{e}^{\alpha x}\right)}{1+\mathrm{e}^{-\alpha x}}-\log \left(1+\mathrm{e}^{\alpha x}\right)\right) \\
& <0
\end{aligned}
$$

Since $\varphi_{k}(x)=\log \left(1+e^{\alpha_{k} x}\right) / \alpha_{k}$, we conclude that if $0<\alpha_{k}<\alpha_{\ell}$ then $\varphi_{k}(x)>\varphi_{\ell}(x)$.

b) For $g_{i}>0$. It is clear that the $h_{i}^{k}: \mathbb{R} \rightarrow \mathbb{R}$ are continuous. To check they are also strictly concave we compute $\left(h_{i}^{k}\right)^{\prime \prime}(x)=h_{i}^{\prime \prime}\left(\varphi_{k}(x)\right) \cdot\left(\varphi_{k}^{\prime}(x)\right)^{2}(x)+h_{i}^{\prime}\left(\varphi_{k}(x)\right) \cdot \varphi_{k}^{\prime \prime}(x)$ where

$$
\begin{aligned}
h_{i}^{\prime}(x)=\frac{g_{i}}{x}-1, \quad \varphi_{k}^{\prime}(x) & =\frac{\mathrm{e}^{\alpha_{k} x}}{1+\mathrm{e}^{\alpha_{k} x}}=\frac{1}{1+\mathrm{e}^{-\alpha_{k} x}}, \\
h_{i}^{\prime \prime}(x) & =-\frac{g_{i}}{x^{2}}, \quad \varphi_{k}^{\prime \prime}(x)=\frac{\alpha_{k} \mathrm{e}^{-\alpha_{k} x}}{\left(1+\mathrm{e}^{-\alpha_{k} x}\right)^{2}},
\end{aligned}
$$

then

$$
\begin{aligned}
\left(h_{i} \circ \varphi_{k}\right)^{\prime \prime}(x)= & -\frac{g_{i}}{\left(\varphi_{k}(x)\right)^{2}} \frac{1}{\left(1+\mathrm{e}^{-\alpha_{k} x}\right)^{2}} \\
& +\left(\frac{g_{i}}{\varphi_{k}(x)}-1\right) \frac{\alpha_{k} \mathrm{e}^{-\alpha_{k} x}}{\left(1+\mathrm{e}^{-\alpha_{k} x}\right)^{2}} \\
= & -\frac{g_{i}\left(1-\mathrm{e}^{-\alpha_{k} x} \log \left(1+\mathrm{e}^{\alpha_{k} x}\right)\right)}{\left(\varphi_{k}(x)\left(1+\mathrm{e}^{-\alpha_{k} x}\right)\right)^{2}} \\
& -\frac{\alpha_{k} \mathrm{e}^{-\alpha_{k} x}}{\left(1+\mathrm{e}^{-\alpha_{k} x}\right)^{2}}<0 \quad \forall x \in \mathbb{R},
\end{aligned}
$$

where we used that $\log (1+y) / y<1$ for $y>0$, i.e., $h_{i}^{k}$ are strictly concave.

Secondly, if $x_{k} \rightarrow x \in \mathbb{R}$ then part a) implies $\varphi_{k}\left(x_{k}\right) \in$ ] $0,+\infty\left[\right.$ and $\varphi_{k}\left(x_{k}\right) \rightarrow \max (0, x)$. From the definition of $h_{i}$ (with $g_{i}>0$ ) it follows that $h_{i}^{k}\left(x_{k}\right)=h_{i}\left(\varphi_{k}\left(x_{k}\right)\right) \rightarrow$ $h_{i}(x)$ for $x>0$ and $h_{i}^{k}\left(x_{k}\right) \rightarrow-\infty$ for $x \leq 0$, i.e. $h_{i}^{k}\left(x_{k}\right) \rightarrow h_{i}(x)$. Finally, since $\max (0, x)<\varphi_{k}(x) \leq$ $\varphi_{0}(x)$ and $\log (x)$ is increasing, it is clear that $h_{i}^{k}(x)=$ $g_{i} \log \varphi_{k}(x)-\varphi_{k}(x) \leq g_{i} \log \varphi_{0}(x)-\max (0, x)=h_{i}^{0}(x)$, while

$$
\begin{aligned}
\lim _{x \rightarrow-\infty} h_{i}^{0}(x) & =\lim _{x \rightarrow-\infty}\left[g_{i} \log \varphi_{0}(x)-\max (0, x)\right] \\
& =\lim _{x \rightarrow-\infty} g_{i} \log \left(\frac{\log \left(1+\mathrm{e}^{\alpha_{0} x}\right)}{\alpha_{0}}\right)=-\infty
\end{aligned}
$$

and

$$
\begin{aligned}
\lim _{x \rightarrow+\infty} h_{i}^{0}(x) & =\lim _{x \rightarrow+\infty}\left[g_{i} \log \varphi_{0}(x)-\max (0, x)\right] \\
& \leq \lim _{x \rightarrow+\infty}\left[g_{i} \log \left(x+\frac{\log (2)}{\alpha_{0}}\right)-x\right] \\
& =-\infty
\end{aligned}
$$

c) For $g_{i}=0$. The same computations as in part b) imply that $h_{i}^{k}: \mathbb{R} \rightarrow \mathbb{R}$ are strictly concave and continuous. To study the limits is a bit different, and we need to consider three cases.

For $x>0$, if $x_{k} \rightarrow x$ then $\varphi_{k}\left(x_{k}\right) \rightarrow x$ and $\log \varphi_{k}\left(x_{k}\right) \rightarrow$ $\log (x) \in \mathbb{R}$, hence

$$
\begin{aligned}
\lim _{k} h_{i}^{k}\left(x_{k}\right) & =\lim _{k}\left[\beta_{k} \log \varphi_{k}\left(x_{k}\right)-\varphi_{k}\left(x_{k}\right)\right] \\
& =0 \cdot \log (x)-x=-x=h_{i}(x) .
\end{aligned}
$$

For $x<0$, if $x_{k} \rightarrow x$, then $\varphi_{k}\left(x_{k}\right)>0$ and

$$
\varphi_{k}\left(x_{k}\right)=\frac{\log \left(1+\mathrm{e}^{\alpha_{k} x_{k}}\right)}{\alpha_{k}} \leq \frac{\mathrm{e}^{\alpha_{k} x_{k}}}{\alpha_{k}},
$$


hence

$$
\begin{aligned}
h_{i}^{k}\left(x_{k}\right) & =\beta_{k} \log \varphi_{k}\left(x_{k}\right)-\varphi_{k}\left(x_{k}\right) \\
& \leq \beta_{k} \log \left(\frac{\mathrm{e}^{\alpha_{k} x_{k}}}{\alpha_{k}}\right)-\varphi_{k}\left(x_{k}\right) \\
& \leq-\beta_{k} \log \left(\alpha_{k}\right)+x_{k} \beta_{k} \alpha_{k}-\varphi_{k}\left(x_{k}\right) \\
& \leq x_{k} \beta_{k} \alpha_{k} \longrightarrow-\infty,
\end{aligned}
$$

i.e., $\lim h_{i}^{k}\left(x_{k}\right)=h_{i}(x)$.

For $x=0$, if $x_{k} \rightarrow 0$ then $0<\varphi_{k}\left(x_{k}\right)<1$ for $k$ large enough, hence

$$
h_{i}^{k}\left(x_{k}\right)=\beta_{k} \log \varphi_{k}\left(x_{k}\right)-\varphi_{k}\left(x_{k}\right)<0
$$

and $\lim \sup _{k} h_{i}^{k}\left(x_{k}\right) \leq 0=h_{i}(0)$. On the other hand, for $x_{k} \rightarrow 0$ with $x_{k} \geq \beta_{k}$ we have

$$
\begin{aligned}
h_{i}^{k}\left(x_{k}\right) & \geq \beta_{k} \log \varphi_{k}\left(\beta_{k}\right)-\varphi_{k}\left(x_{k}\right) \\
& =\beta_{k} \log \left(\frac{\log \left(1+\mathrm{e}^{\alpha_{k} \beta_{k}}\right)}{\alpha_{k}}\right)-\varphi_{k}\left(x_{k}\right) \\
& \geq \beta_{k} \log \left(\frac{\log \left(\mathrm{e}^{\alpha_{k} \beta_{k}}\right)}{\alpha_{k}}\right)-\varphi_{k}\left(x_{k}\right) \\
& =\beta_{k} \log \left(\beta_{k}\right)-\varphi_{k}\left(x_{k}\right) \rightarrow 0,
\end{aligned}
$$

hence $\liminf _{k} h_{i}^{k}\left(x_{k}\right) \geq 0=h_{i}(0)$.

To prove that the $h_{i}^{k}$ 's are bounded by $h_{i}^{0}$ we study three cases. If $x<0$ then $h_{i}^{k}(x) \leq x \beta_{k} \alpha_{k}$ as above, and

$$
h_{i}^{k}(x) \leq x \beta_{k} \alpha_{k} \leq \kappa_{0} x=h_{i}^{0}(x) .
$$

If $0 \leq x \leq \varphi_{0}^{-1}(1)$, we use that $h_{i}^{k}(x) \leq \beta_{k} \log \left(\beta_{k}\right)-\beta_{k}$ (for all $x \in \mathbb{R}$ ) and $\beta_{k} \leq \mathrm{e}$, hence $h_{i}^{k}(x) \leq 0 \leq \kappa_{o} x=$ $h_{i}^{0}(x)$ for $x \in\left[0, \varphi_{0}^{-1}(1)\right]$.

For $\varphi_{0}(x)>1$, we have $0<x<\varphi_{k}(x) \leq \varphi_{0}(x)$ and $\log \varphi_{0}(x)>0$, hence

$$
\begin{aligned}
h_{i}^{k}(x) & =\beta_{k} \log \varphi_{k}(x)-\varphi_{k}(x) \\
& \leq \beta_{k} \log \varphi_{0}(x)-x \\
& \leq \beta_{0} \log \varphi_{0}(x)-x=h_{i}^{0}(x) .
\end{aligned}
$$

And $h_{i}^{0}$ is coercive since (using some calculations from above)

$$
\begin{aligned}
& \lim _{x \rightarrow-\infty} h_{i}^{0}(x)=\lim _{x \rightarrow-\infty} \kappa_{0} x=-\infty, \text { while } \\
& \lim _{x \rightarrow+\infty} h_{i}^{0}(x) \leq \lim _{x \rightarrow+\infty} \beta_{0} \log \left(x+\frac{\log (2)}{\alpha_{0}}\right)-x=-\infty .
\end{aligned}
$$

\section{REFERENCES}

[1] F. Natterer, The Mathematics of Computerized Tomography, ser. Classics in Applied Mathematics. SIAM, 2001.

[2] L. A. Shepp and Y. Vardi, "Maximum likelihood reconstruction for emission tomography," IEEE Trans. Med. Imag., vol. 1, no. 2, pp. 113122, 1982.

[3] K. Lange and R. Carson, "EM reconstruction algorithms for emission and transmission tomography," J. Comput. Assist. Tomogr., vol. 8, no. 2, pp. 306-316, 1984

[4] H. M. Hudson and R. S. Larkin, "Accelerated image reconstruction using ordered subsets of projection data," IEEE Trans. Med. Imag., vol. 13 , no. 4, pp. 601-609, 1994.
[5] A. R. De Pierro, "A modified expectation maximization algorithm for penalized likelihood estimation in emission tomography," IEEE Trans. Med. Imag., vol. 14, no. 1, pp. 132-137, 1995.

[6] P. J. Green, "Bayesian reconstructions from emission tomography data using a modified EM algorithm," IEEE Trans. Med. Imag., vol. 9, no. 1, pp. 84-93, 1990.

[7] S. Ahn and J. A. Fessler, "Globally convergent image reconstruction for emission tomography using relaxed ordered subsets algorithms," IEEE Trans. Med. Imag., vol. 22, no. 5, pp. 613-626, 2003.

[8] M. Soussan, F. Orlhac, M. Boubaya, L. Zelek, M. Ziol, V. Eder, and I. Buvat, "Relationship between tumor heterogeneity measured on FDGPET/CT and pathological prognostic factors in invasive breast cancer," PLOS One, vol. 9, no. 4, p. e94017, 2014.

[9] M. Hatt, F. Tixier, L. Pierce, P. E. Kinahan, C. Cheze-Le Rest, and D. Visvikis, "Characterization of PET/CT images using texture analysis the past, the present... any future?" Eur. J. Nucl. Med. Mol. Imag., vol. 44, no. 1, pp. 151-165, 2017.

[10] T. Carlier, K. P. Willowson, E. Fourkal, D. L. Bailey, M. Doss, and M. Conti, "Y90-PET imaging: Exploring limitations and accuracy under conditions of low counts and high random fraction," Med. Phys., vol. 42, no. 7, pp. 4295-4309, 2015.

[11] K. Erlandsson, D. Visvikis, W. Waddington, I. Cullum, P. Jarritt, and L. Polowsky, "Low-statistics reconstruction with AB-EMML," in IEEE Nucl. Sci. Symp. Conf. Rec., 2000.

[12] G. Wang and J. Qi, "Low-count PET image reconstruction using sinogram shifting (abstract only)," in IEEE Nucl. Sci. Symp. Conf. Rec., 2012.

[13] J. Nuyts, S. Stute, K. Van Slambrouck, F. van Velden, R. Boellaard, and C. Comtat, "Maximum-likelihood reconstruction based on a modified Poisson distribution to reduce bias in PET," in IEEE Nucl. Sci. Symp. Conf. Rec., 2011.

[14] K. Van Slambrouck, S. Stute, C. Comtat, M. Sibomana, F. H. van Velden, R. Boellaard, and J. Nuyts, "Bias reduction for low-statistics PET: Maximum likelihood reconstruction with a modified Poisson distribution," IEEE Trans. Med. Imag., vol. 34, no. 1, pp. 126-136, 2015.

[15] H. Lim, Y. K. Dewaraja, and J. Fessler, "A PET reconstruction formulation that enforces non-negativity in projection space for bias reduction in Y-90 imaging," Phys. Med. Biol., vol. 63, no. 3, 2018.

[16] S. Boyd, N. Parikh, E. Chu, B. Peleato, and J. Eckstein, "Distributed optimization and statistical learning via the alternating direction method of multipliers," Foundations \& Trends in Machine Learning, vol. 3, no. 1, pp. 1-122, 2010.

[17] A. Bousse and M. Courdurier, "Hypo-convergence for pet reconstruction with non-negativity constraints in projection space (abstract only)," in IEEE Nucl. Sci. Symp. Med. Imag. Conf. Rec., 2018.

[18] H. Erdoğan and J. A. Fessler, "Monotonic algorithms for transmission tomography," IEEE Trans. Med. Imag., vol. 18, no. 9, pp. 801-814, 1999.

[19] —_ "Ordered subsets algorithms for transmission tomography," Phys. Med. Biol., vol. 44, no. 11, pp. 2835-2851, 1999.

[20] Y.-J. Tsai, A. Bousse, M. J. Ehrhardt, C. W. Stearns, S. Ahn, B. H. Hutton, S. Arridge, and K. Thielemans, "Fast quasi-newton algorithms for penalized reconstruction in emission tomography and further improvements via preconditioning," IEEE Trans. Med. Imag., vol. 37, no. 4, pp. $1000-1010,2018$

[21] E. Ghadimi, A. Teixeira, I. Shames, and M. Johansson, "Optimal parameter selection for the alternating direction method of multipliers (ADMM): Quadratic problems," IEEE Trans. Automat. Contr., vol. 60, no. 3, pp. 644-658, 2015.

[22] Z. Xu, M. Figueiredo, and T. Goldstein, "Adaptive ADMM with spectral penalty parameter selection," in Proc. of the 20th Int. Conf. on Artificial Intelligence and Statistics, ser. Proc. of Machine Learning Research, vol. 54. PMLR, 2017, pp. 718-727.

[23] J. Nocedal and S. J. Wright, Numerical Optimization, 2nd ed. Springer, 2006.

[24] C. Zhu, R. H. Byrd, P. Lu, and J. Nocedal, "Algorithm 778: L-BFGS-B Fortran subroutines for large-scale bound-constrained optimization," $A C M$ T. Math. Software, vol. 23, no. 4, pp. 550-560, 1997.

[25] N. A. Karakatsanis, E. Fokou, and C. Tsoumpas, "Dosage optimization in positron emission tomography: State-of-the-art methods and future prospects," Am. J. Nucl. Med. Mol. Imaging, vol. 5, no. 5, pp. 527-547, 2015.

[26] W. P. Segars, G. Sturgeon, S. Mendonca, J. Grimes, and B. M. Tsui, "4D XCAT phantom for multimodality imaging research," Med. Phys, vol. 37, no. 9, pp. 4902-4915, 2010.

[27] R. T. Rockafellar and R. J.-B. Wets, Variational Analysis, 1st ed. SpringerVerlag Berlin Heidelberg, 1998. 